\title{
Kombinative Betrachtung von Naturmessung, physikalischer und numerischer Modellierung des Geschiebetransports an großen alpinen Wasserfassungen
}

\author{
Manuel Plörer · Johann Neuner · Stefan Achleitner · Markus Aufleger
}

Online publiziert: 6. März 2017

(c) Der/die Autor(en) 2017. Dieser Artikel ist eine Open-Access-Publikation.

Zusammenfassung Die Tiroler Wasserkraft AG (TIWAG) plant im Zuge des Ausbaus des Kraftwerks Kaunertal die Errichtung von zwei großen Wasserfassungen an Sperrenbauwerken an der Gurgler Ache und an der Venter Ache im Ötztal/Tirol. Hinsichtlich einer effektiven Geschiebebewirtschaftung sollen regelmäßige Spülungen der Stauräume durchgeführt werden. Die in diesem Zusammenhang stehenden technischen Fragestellungen wurden am Arbeitsbereich Wasserbau der Universität Innsbruck durch Untersuchungen an einem physikalischen Modellversuch, mit numerischen Berechnungen und mit Naturmessungen bearbeitet. Die dargestellte Untersuchungsmethodik zeigt die Kalibrierungs- und Validierungsschritte für die Simulation der Verlandungsvorgänge, der Stauraumspülungen und der Spülungen der unmittelbaren Unterwasserstrecke mit dem 2D-numerischen Geschiebetransportmodell Hydro_GS-2D. Die Kalibrierung der numerischen Simulationen wurde mithilfe der Erkenntnisse und der Ergebnisse aus einem physikalischen Modellversuch für die Wasserfassung Gurgler Ache durchgeführt. Die Simulationen erfolgten mit drei verschiedenen Modelltypen, welche für eine schrittweise Übertragung der relevanten Parameter im Modellmaßstab bis hin zum Naturmaßstab verwendet

\section{M. Plörer $(\bowtie)$ •}

Assoz.-Prof. DI Dr. S. Achleitner .

Univ.-Prof. Dr.-Ing. M. Aufleger Arbeitsbereich Wasserbau Institut für Infrastruktur, Universität Innsbruck, Technikerstraße 13, 6020 Innsbruck, Österreich

manuel.ploerer@uibk.ac.at

\section{Dr.-Ing. J. Neuner}

TIWAG - Tiroler Wasserkraft AG, Eduard-Wallnöfer-Platz 2, 6020 Innsbruck, Österreich

johann.neuner@tiwag.at wurden. Eine zusätzliche Validierung der gewählten Einstellungen konnte mit der numerischen Modellierung des Spülvorganges an einer bereits bestehenden Anlage, wo umfangreiche Naturmessungen als Berechnungsgrundlage vorliegen, erreicht werden. Das Ineinandergreifen der drei Modellteile - Numerik, physikalischer Modellversuch und Naturmessung - ermöglicht eine wissenschaftliche Absicherung der Berechnungsergebnisse in den numerischen Simulationen und eine Evaluierung von Modellierungsproblemen und Unsicherheiten. Mit den gewählten Ansätzen können die Verlandungsvorgänge im Stauraum und die Spülungen in der Unterwasserstrecke mithilfe von 2D-numerischen Simulationen sehr gut abgebildet werden. Daneben zeigt sich, dass der Geschiebetransport während der Stauraumspülung mit etablierten Transportparametern um den Faktor 2 bis 3 unterschätzt wird, und damit eine entsprechende Anpassung notwendig wird.

Combined assessment of bedload transport at major alpine water intakes: field study, numerical and physical modeling

Abstract As part of the Kaunertal hydro power plant expansion, the Tiroler Wasserkraft AG (TIWAG) is planning to construct two large front-type water intakes at the Gurgler Ache and at the Venter Ache in Ötztal, Tyrol. To ensure an effective bed-load transport management, the connected reservoirs should be regularly flushed. The Department of Hydraulic Engineering at the University of Innsbruck has investigated the technical aspects of this process using a physical model, numerical simulations and field measurements.

The research methods presented show the calibration and validation steps for the simulations of the reservoir sedimentation and flushing pro- cesses, as well as flushing in the area immediately downstream using the 2Dhydrodynamical simulation software Hydro_GS-2D. The numerical models were calibrated on the basis of results and findings from the physical model study carried out for the water intake Gurgler Ache. The simulations were based on three different model types, which were used to extrapolate the relevant parameters from model to fullscale on a step-by-step basis. In addition, a validation of the selected parameters was conducted by a numerical simulation of the flushing process at an already existing water intake structure which is similar to the planned structures. Herein extensive field data is available.

The integration of the three model parts - field study, numerical and physical modeling - allows to validate the results of the numerical simulations and to evaluate modeling problems and uncertainties. Employing the selected approaches shows that the sedimentation processes in the reservoir and flushing processes downstream can be modeled using two-dimensional simulations. It is also revealed that simulations based on established transport parameters underestimate the bed-load transport during the reservoir flushing by a factor of 2 to 3 , making a corresponding adjustment necessary.

\section{Einleitung}

1.1 Kurzbeschreibung der projektierten Wasserfassungen Gurgler Ache und Venter Ache

Das Speicherkraftwerk Kaunertal ist die größte Anlage der Kraftwerksgruppe der Tiroler Wasserkraft AG (TIWAG). Der Nutzinhalt des Speichers Gepatsch beträgt ca. 139 Mio. $\mathrm{m}^{3}$ und die jährliche Einzugsfracht umfasst durchschnittlich 323 Mio. $\mathrm{m}^{3}$. Die Regeljahreserzeugung umfasst $660 \mathrm{GWh}$. Neben dem natürli- 

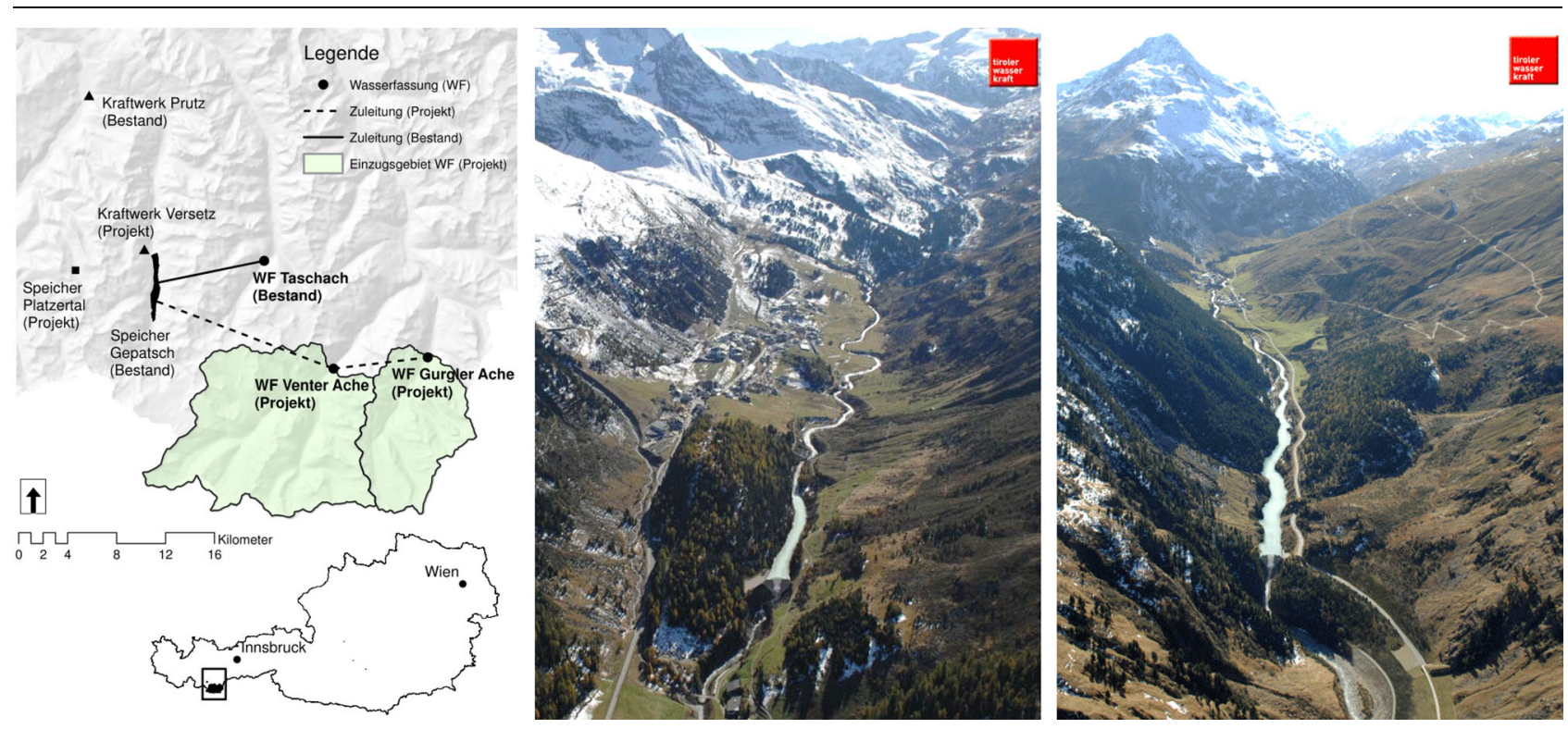

Abb. 1 links: Projektgebiet; Mitte: Fotomontage WF Gurgler Ache; rechts: Fotomontage WF Venter Ache (TIWAG 2016)

chen Einzugsgebiet besitzt die Anlage auch mehrere Zuleitungen, darunter auch die in der Modellierung bearbeitete Zuleitung von der Wasserfassung Taschachbach im Pitztal. Die gesamte Einzugsgebietsgröße aus dem natürlichen und dem durch Zuleitungen erfassten Einzugsgebiet ergibt $279 \mathrm{~km}^{2}$. Im Zuge der Erweiterung des Kraftwerks sind zwei neue Wasserfassungen an Sperrenbauwerken an der Gurgler Ache und an der Venter Ache im Ötztal/Tirol geplant (Abb. 1). Im Hinblick auf einen wartungsarmen Betrieb der Anlagen soll die Geschiebebewirtschaftung an den Wasserfassungen mit regelmäßigen Spülungen der zugehörigen Stauräume erfolgen.

Die zwei projektierten Wasserfassungen „Gurgler Ache“ und „Venter Ache" sollen in einer Höhe von ungefähr $1840 \mathrm{~m}$. ü. A. angeordnet werden. Dabei ergeben sich Stauhaltungen von jeweils ca. $60000 \mathrm{~m}^{3}$. Die Einzugsgebiete für die Wasserfassungen besitzen eine Größe von $79.7 \mathrm{~km}^{2}$ an Gurgler Ache und $179.7 \mathrm{~km}^{2}$ an der Venter Ache. Die maximale Entnahme soll $29 \mathrm{~m}^{3} / \mathrm{s}$ (Gurgler Ache) bzw. $50 \mathrm{~m}^{3} / \mathrm{s}$ (Venter Ache) umfassen, womit sich spezifische Entnahmen von $364 \mathrm{l} / \mathrm{s} / \mathrm{km}^{2}$ bzw. $278 \mathrm{l} / \mathrm{s} / \mathrm{km}^{2}$ ergeben. Die Hochwasserentlastungen werden durch feste Überfälle auf den Bogenstaumauern gewährleistet. Daneben steht an beiden Bauwerken eine Grundablassöffnung mit Drucksegment zur Verfügung, mit welcher der geplante Spülvorgang gesteuert werden kann. Die bereits be- stehende Anlage „Taschachbach“ basiert auf den gleichen Konstruktionsgrundsätzen, ist jedoch hinsichtlich der maximalen Entnahme und des Stauraumvolumens deutlich kleiner. Trotz der Unterschiede in den Bauwerksdimensionen bestehen nachfolgende Gemeinsamkeiten der drei Anlagen, welche zur Wahl der in diesem Beitrag dargestellten Bearbeitungsmethodik führte:

a) Charakteristik des Einzugsgebietes: Topografie, Hydrologie, Vegetation, Vergletscherung,

b) Bauwerkskonstruktion: Staumauer, Hochwasserentlastung, steuerbares Grundablassdrucksegment,

c) Kornzusammensetzung des transportierten Geschiebes, Sohlstrukturen im natürlichen Gerinne,

d) Gefälleverhältnisse in der Zulaufstrecke und in der Unterwasserstrecke.

\subsection{Zielsetzung der Untersuchungen}

Mithilfe eines physikalischen Modellversuchs, numerischer Simulationen und Naturmessungen soll der Geschiebetransport an den geplanten Wasserfassungen während der Verlandungsvorgänge und der Spülvorgänge eingehend untersucht werden. Damit kann frühzeitig in der Planungsphase vorhandenes Optimierungspotenzial erkannt werden. Zusätzlich kann gezeigt werden, ob die gewählte Methode des Geschiebemanagements langfristig zu den notwendigen Ergebnissen führt, oder ob andere, begleitende Maßnahmen notwendig werden. Dabei wurden hinsichtlich des geplanten Regelbetriebs Stauraumbelastungen in der Größenordnung von einer halben, mittleren jährlichen Geschiebefracht (1/2 JGF) modelliert. Der Fokus der Untersuchungen liegt auf der Betrachtung der Belastungen infolge von Hochwasserereignissen, wo mit hohen Abflüssen und großen, grobkörnigen Geschiebeeinträgen in kurzer Zeit zu rechnen ist. Dabei soll zum einem geklärt werden, ob große Feststofffrachten vom Stauraum aufgenommen werden können, ohne den Betrieb der Anlage zu beeinträchtigen. Zudem muss gezeigt werden, dass eine Spülung dieser Geschiebebelastungen sowohl im Stauraum als auch in der Unterwasserstrecke möglich ist.

\section{Methodik}

\subsection{Modellkonzept}

Aufgrund der vorherrschenden Rahmenbedingungen und der definierten Zielsetzungen entwickelte der Arbeitsbereich Wasserbau der Universität Innsbruck in Zusammenarbeit mit der Tiroler Wasserkraft AG eine Bearbeitungsmethodik, um eine effektive und wissenschaftlich belastbare Untersuchung der Verlandungs- und Spülprozesse an den zwei geplanten Wasserfassungen sowie an der bereits bestehenden Anlage Taschachbach durchzuführen (Abb. 2). 


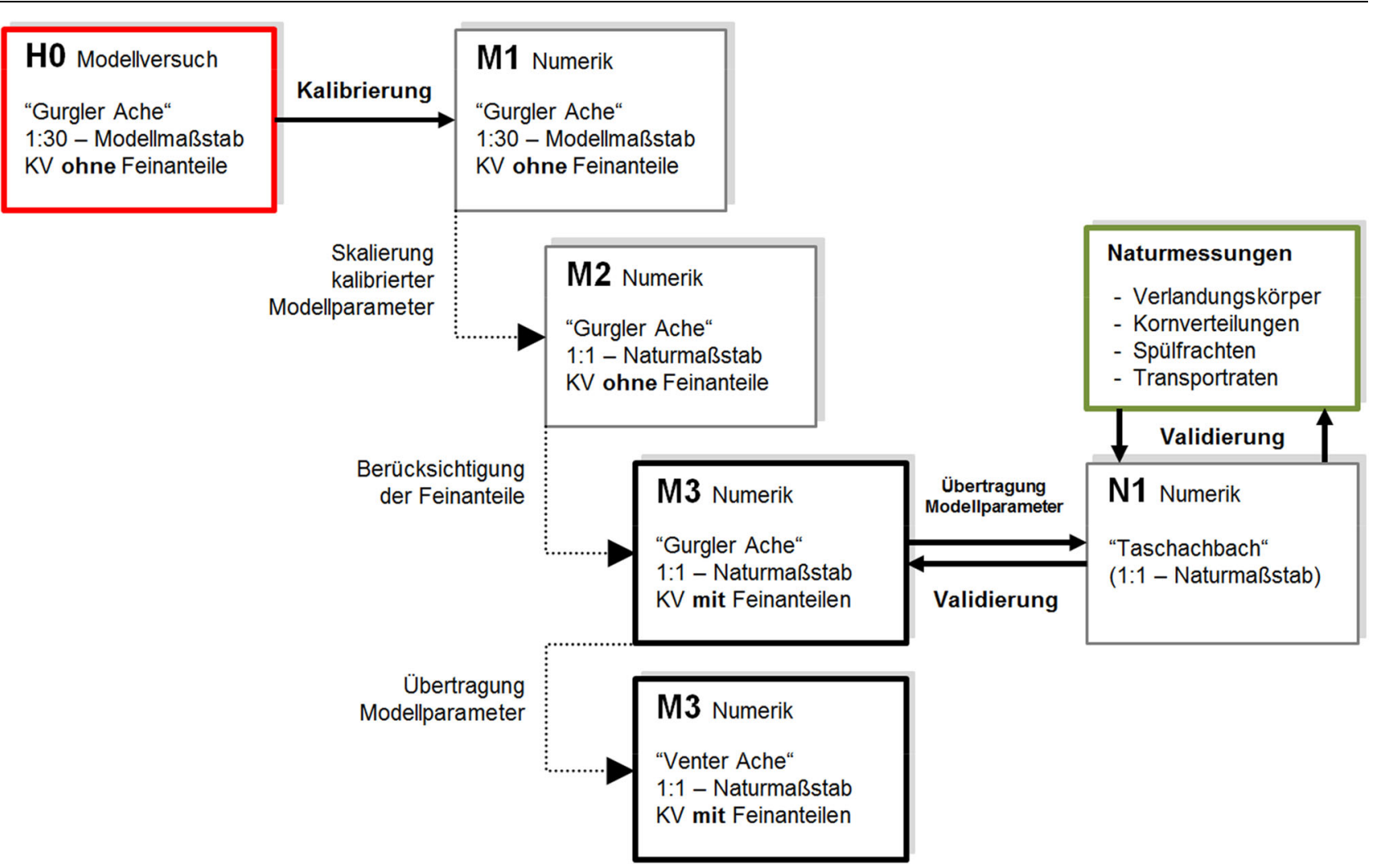

Abb. 2 Modellierungskonzept (KV=Kornverteilung; Definition Feinanteile nach Abschnitt 2.2)

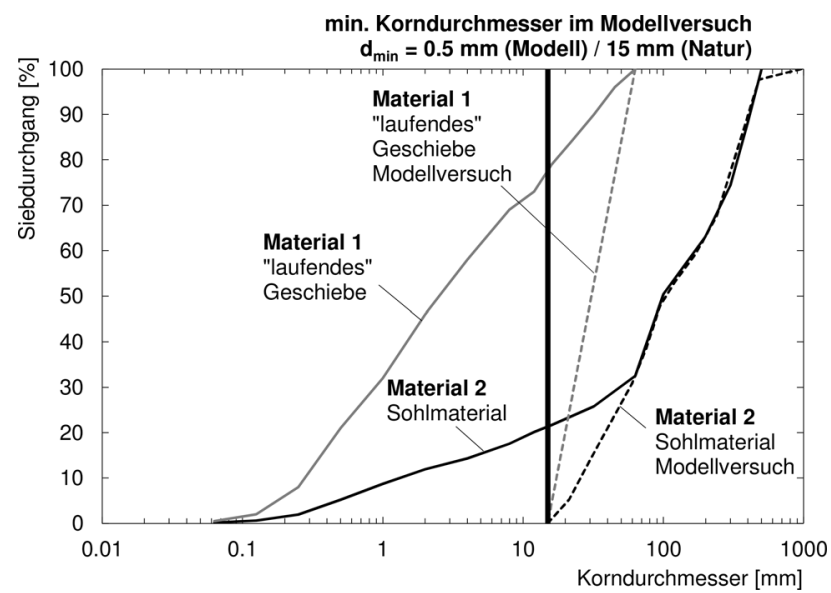

Abb. 3 Vergleich Kornverteilungen (Material 1,2) des Geschiebes nach Naturmessungen und im Modellversuch (Naturmaßstab)

In einem ersten Schritt wurde die geplante Wasserfassung an der Gurgler Ache im Wasserbaulabor der Universität Innsbruck mit einem physikalischen Modellversuch im Maßstab 1:30 untersucht (Kapitel 3). Die Ergebnisse wurden verwendet um numerische Simulationen im Modellmaßstab zu kalibrieren (Kapitel 4). Die verwendeten maßstabsabhängigen Parameter wurden dann für Simulationen im Natur- maßstab skaliert. Schließlich erfolgte eine Übertragung der Parameter des kalibrierten Modells "Gurgler Ache“ zur numerischen Simulation der Anlage „Venter Ache“. Die numerische Simulation einer realen Spülung an der Wasserfassung „Taschachbach“ dient der Validierung der gewählten Modelleinstellungen und führt damit zu einer Absicherung der vorangegangen Parameterwahl (Kapitel 5).
2.2 Beschreibung der modellbedingten Rahmenbedingungen und Einschränkungen

\subsubsection{Reiner Geschiebetransport}

Die Simulationen der Verlandungsund Spülvorgänge basieren auf dem Ansatz eines reinen Geschiebetransports. Auf eine Berücksichtigung der Schwebstoffanteile wird aufgrund des sehr kleinen Verhältnisses von Stauraumvolumen $\mathrm{zu}$ Jahreszufluss (Speicherkennzahl $<0.001$ ) verzichtet. Diese Annahme bezieht sich u. a. auf die Untersuchungen von Brune (1953), wonach der zurückgehaltene Schwebstoffanteil im Verlandungskörper bei Stauräumen mit Kennzahlen kleiner als 0.003 gegen Null geht. Vereinfachend kann für die Bearbeitung davon ausgegangen werden, dass Schwebstoffe teilweise eingezogen werden, über die Mauer transportiert werden, oder im Falle einer Rückhaltung bei einer Spülung wieder als Schwebstoffe im Wasserkörper transportiert werden und es damit zu keiner Absetzung in der Unterwasserstrecke kommt. 


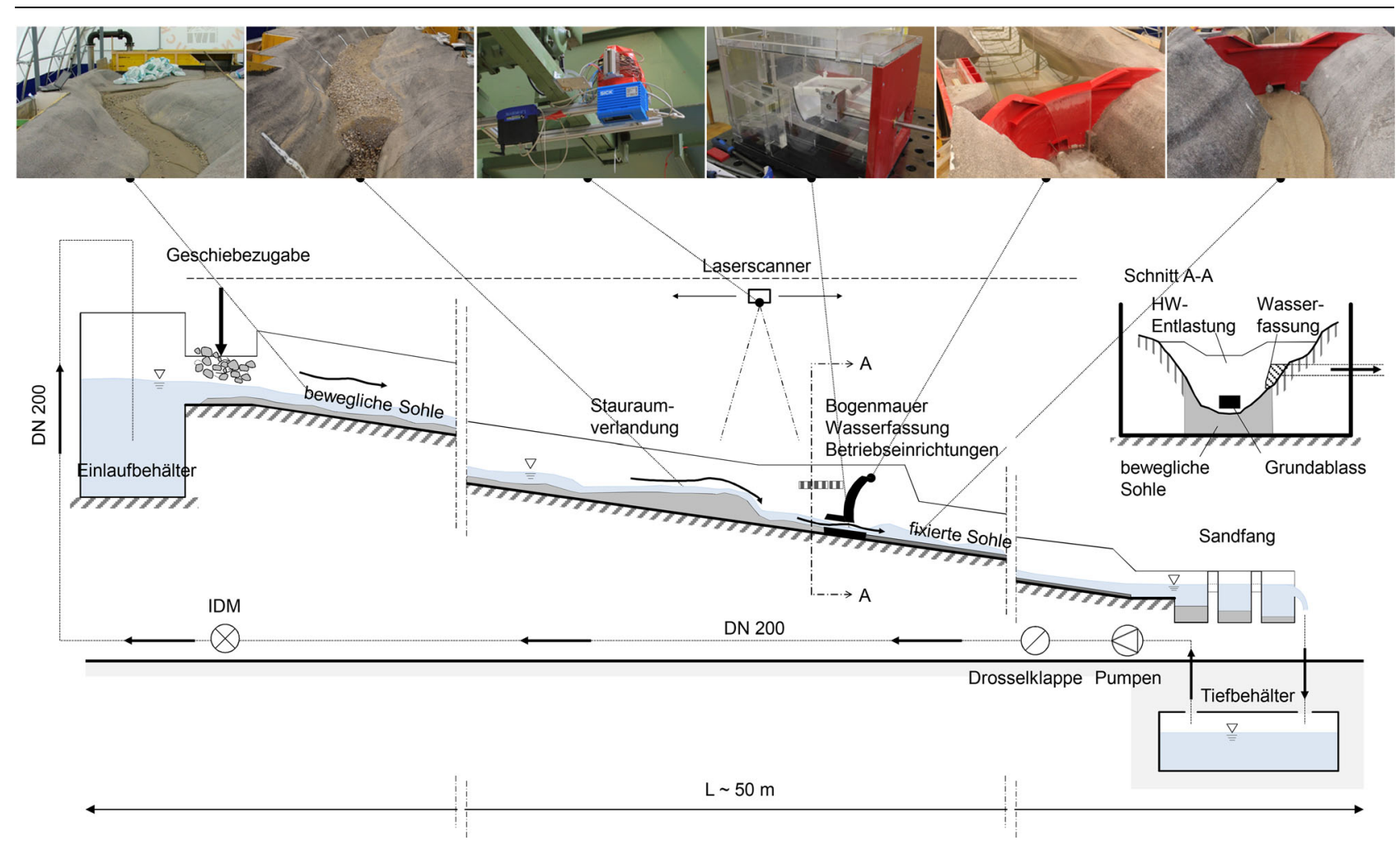

Abb. 4 Systemskizze physikalischer Modellversuch Wasserfassung Gurgler Ache

\subsubsection{Zusammensetzung der Geschiebefracht}

Die Kornverteilungen des Geschiebematerials wurden mit Naturmessungen (Linienzahlanalysen, Baggerschürfe) im Projektgebiet bestimmt. Daraus wurde für den physikalischen Modellversuch und für die numerischen Berechnungen für das transportierte Geschiebe folgende Regelung festgelegt (AB Wasserbau 2012; AB Wasserbau 2015a; Plörer et al. 2013a):

- Abflussbereich $Q<60 \mathrm{~m}^{3} / \mathrm{s}$ : kein Aufreißen der Deckschicht; feine Kornzusammensetzung entsprechend Geschiebematerial 1 nach Abb. 3.

- Abflussbereich $\mathbf{Q}>60 \mathrm{~m}^{3} / \mathrm{s}$ : Aufreißen der Deckschicht; grobe Kornzusammensetzung entsprechend Geschiebematerial 2 nach Abb. 3.

Der Schwellenwert wurde auf Basis der hydrologischen Kennwerte an der Gurgler Ache bestimmt. Im Bereich der geplanten Wasserfassung ist das 5-jährliche Hochwasser (HQ5) mit einem Spitzenabfluss von ca. $60 \mathrm{~m}^{3} / \mathrm{s}$ definiert. Für die Bestimmung der Geschiebezusammensetzung geht man von einer stabilen Deckschicht bis zu diesem Abfluss aus. Liegt der Abfluss über
$60 \mathrm{~m}^{3} / \mathrm{s}$, beginnt die Deckschicht aufzureißen und es wird verstärkt grobes Material transportiert. Diese Annahme ermöglicht die Berücksichtigung grober Geschiebeeinträge während hoher Abflüsse und den Eintrag von Material mit geringerer Korngröße während eines über das Jahr verteilten, quasi-kontinuierlichen Verlandungsprozesses. Diese vereinfachten Zusammenhänge wurden auch für die Untersuchungen an der Venter Ache übernommen.

\subsubsection{Mindestkorndurchmesser}

Die Kornverteilungen des Geschiebematerials wurden für die Experimente im Labor modifiziert und vergröbert. Der Grund dafür liegt in der notwendigen Einhaltung von Mindestkorngrößen von etwa $0.5 \mathrm{~mm}$ im hydraulischen Versuchswesen, womit eine Übertragung der Modellergebnisse in die Natur möglich wird. In Anbetracht des Modellmaßstabes von 1:30 können nur Korngrößen bis $15 \mathrm{~mm}$ berücksichtigt werden. Um die Massenbilanz nicht zu verändern, wurden Sedimente mit einem Korndurchmesser kleiner als $15 \mathrm{~mm}$ der feinsten darstellbaren Fraktion zugeordnet (Abb. 3). Mit dieser Annahme ist die Kornzusammensetzung des Geschiebes deutlich gröber als in Natur, womit die ermittelten Transportleistungen im Modell tendenziell geringer sein dürften als in der Natur und hinsichtlich einer Bewertung der Spülleistungen auf der sicheren Seite liegen.

In diesem Zusammenhang wird von einer „Kornverteilung ohne Feinanteile“ bzw. einer „abgeschnittenen Kornverteilung“ gesprochen. Diese abgeschnittene Kornverteilung wurde vom Modellversuch in das numerische Modell M1 übernommen (Abb. 2). Die Parameter des kalibrierten Modells M1 im Maßstab 1:30 wurden skaliert und auf die numerische Simulation in Naturmaßstab übertragen. Hier wird zwischen zwei Modelltypen, M2 und M3, unterschieden. Bei M2 handelt es sich um eine Simulation im Naturmaßstab mit der skalierten Kornverteilung ohne Feinanteile, entsprechend dem Modellversuch. Diese Feinanteile werden hingegen in Modelltyp M3 berücksichtigt. Mit dieser Vorgehensweise können neben den Skalierungseffekten auch mögliche Einflüsse der Feinanteile in der Geschiebezusammensetzung auf den Verlandungs- und Spülprozess in den Berechnungen dargestellt werden. 
Tab. 1 Bemessungsgeschiebefrachten am Modelleinlauf (Feststoffvolumen)

\begin{tabular}{|l|l|l|} 
& $\begin{array}{l}\text { Gurgler } \\
\text { Ache }\end{array}$ & $\begin{array}{l}\text { Venter } \\
\text { Ache }\end{array}$ \\
\hline 1/2 JGF & $4.150 \mathrm{~m}^{3}$ & $8.960 \mathrm{~m}^{3}$ \\
\hline Aug 1987 & $22.614 \mathrm{~m}^{3}$ & $60.572 \mathrm{~m}^{3}$ \\
\hline Sep 1999 & $16.345 \mathrm{~m}^{3}$ & $21.584 \mathrm{~m}^{3}$
\end{tabular}

\section{3 Übertragung der Modellparameter} zur Wasserfassung Venter Ache

Aufgrund der beschriebenen Rahmenbedingungen in Abschnitt 2.1 (ähnliche Bauwerkskonstruktionen, Stauraumgeometrie, Einzugsgebietscharakteristika, hydrologische Verhältnisse, Gefälleverhältnisse, Kornzusammensetzung des transportierten Geschiebes) wurde für die zweite geplante Wasserfassung Venter Ache auf einen weiteren Modellversuch verzichtet und die Modellparameter vom Modelltyp M3 „Gurgler Ache" auf das numerische Modell Modelltyp M3 „Venter Ache“ übertragen. Mit dieser Methode soll gewährleistet werden, dass auch ohne direkte Kalibriergrößen aus einem Modellversuch für die Wasserfassung Venter Ache die Ergebnisse der numerischen Berechnungen für belastbare Aussagen herangezogen werden können.

\subsection{Allgemeine Problemstellungen und Lösungsansätze}

Für die Erläuterung der Modellierungsprobleme ist kurz auf die Ergebnisse vorzugreifen. Für die Verlandungsvorgänge und für die Spülungen in der Unterwasserstrecke konnten innerhalb weniger Kalibrierschritte sehr gute Übereinstimmungen mit den Resultaten im Modellversuch erzielt werden. Hingegen zeigten die ersten Ergebnisse der numerischen Berechnungen der Stauraumspülungen einen stark verminderten Transport im Vergleich zum Modellversuch. Die Anpassung der räumlichen und zeitlichen Diskretisierung und von verschiedenen Modellparametern ergaben nur eine beschränkte Steigerung des Transportes. Auch eine Reduktion der dimensionslosen kritischen Schubspannung führte nicht zu der notwendigen Erhöhung der Transportraten. Damit wurde in einem letzten Schritt versucht, den Geschiebetransport durch eine lineare Skalierung zu verstärken. Da diese Skalierung eine Abweichung $\mathrm{zu}$ etablierten und bekannten Literaturwerten darstellt, wurde im Sinne einer wissenschaftlich belastbaren Begründung der Modelleinstellungen ein zusätzlicher Validierungsschritt durchgeführt. Basierend auf Daten der realen Spülung an der Wasserfassung Taschachbach wurde der Spülvorgang mit dem verwendeten 2D-numerischen Ansatz „nachmodelliert“. Diese Ergebnisse zeigen, dass die Verwendung eines Skalierfaktors eine vertretbare Methode ist, um den Transport zu steuern. Mit dieser Maßnahme können nahezu idente Transportraten im Modellversuch und in der 2D-numerischen Simulation bestimmt werden.

\section{Physikalischer Modellversuch Wasserfassung Gurgler Ache (H0)}

\section{1 Überblick Modellversuch}

Der Modellversuch Gurgler Ache basiert auf den Froude'schen Modellgesetz. Dieser Ansatz im wasserbaulichen Versuchswesen für die Modellierung der Fließzustände an offenen Gerinnen beschreibt, dass die Froude'sche Zahl bei den Strömungsvorgängen im Modellversuch gleich groß ist wie in Natur. Mit diesem Zusammenhang lassen sich die Maßstabzahlen für andere physikalische Größen bestimmen. Basierend auf einem geometrischen Maßstab von 1:30, ergeben sich damit ein Durchflussmaßstab von 1:4930 und ein Zeitmaßstab von 1:5.48. Das bedeutet, dass die Prozesse im Modellversuch der Wasserfassung Gurgler Ache in etwa 5.5-mal so schnell ablaufen wie in der Natur.

Es wurden ca. $800 \mathrm{~m}$ von der $\mathrm{Zu}$ laufstrecke und vom Stauraum sowie rund $600 \mathrm{~m}$ von der Strecke nach dem Sperrenbauwerk modelliert (Abb. 4). Im Bereich der Zulaufstrecke und im Stauraum wurde die Modellsohle beweglich ausgeführt und im Bereich der Unterwasserstrecke fixiert. Der Zulauf zum Modell konnte mit einer vordefinierten Ganglinie gesteuert werden und erfolgte über einen Einlaufbehälter. Am oberen Modellrand fand die manuelle Geschiebezugabe statt. Das Drucksegment in der Grundablassöffnung wurde beweglich ausgeführt, womit der Absenkvorgang im Stauraum gelenkt werden konnte. Am unteren Modellende wurde ein Sandfang angeordnet, in dem sich das aus dem Modell transportierte Geschiebe absetzte und so ein Einzug von Sedimenten in den Tiefbehälter vermieden wurde ( $A B$ Wasserbau
2012; Plörer et al. 2013a; Plörer et al. 2013b).

Die Abflussganglinien und der damit verbundene Geschiebeeintrag für die untersuchten Stauraumbelastungen (siehe 3.2) stammen aus einer mit einem hydrologischen Modell kombinierten Geschiebetransportberechnung nach Gems et al. (2009) und Gems (2011). Der Zufluss wurde entsprechend der ermittelten Ganglinien ins Modell geführt und das transportierte Geschiebe manuell laut der berechneten Transportraten zugegeben, womit ein entsprechender Verlauf des Verlandungsvorganges realistisch abgebildet werden konnte.

Die Ermittlung der Verlandungsvolumen, der Spülfrachten und Transportraten erfolgte durch eine Volumenbilanzierung. Dazu wurde zunächst vor jedem Versuch die Ausgangssohle mittels Laserscanning vermessen und schließlich definierte Zwischenzustände während der Versuche sowie der Endzustand der Sohle bestimmt. Durch den Vergleich der Vorher/Nachher-Situation konnten Auflandungshöhen, Erosionstiefen und die zeitabhängigen Transport- bzw. die Spülraten ermittelt werden.

\subsection{Untersuchte Stauraumbelastungen}

Der geplante Betrieb der Anlagen sieht jährlich zwei Spülungen der Stauräume vor. Aus diesem Grund ist zunächst eine Stauraumbelastung mit einer halben jährlichen Geschiebefracht $\left(1 / 2 \mathrm{JGF}=4150 \mathrm{~m}^{3}\right.$ Feststoffvolumen am geplanten Standort der Wasserfassung Gurgler Ache, Tab. 1) von großem Interesse. Eine Belastung infolge von Hochwassereinträgen wurde mit zwei verschiedenen historischen Hochwasserereignissen, bezogen auf einen Ereigniszeitraum von $72 \mathrm{~h}$, untersucht. Das Hochwasser vom August 1987 ist bestimmt durch eine große kumulative Wasserfracht und eine Geschiebefracht in der Größenordnung von ca. $275 \%$ der Jahresgeschiebefracht $\left(22614 \mathrm{~m}^{3}\right)$. Der Spitzenabfluss ist äquivalent $\mathrm{zu}$ der Größe eines 10-jährlichen Hochwassers (HQ10) (Abb. 5). Das Hochwasserereignis vom September 1999 ist geprägt durch eine Ganglinie mit starkem Anstieg und Rückgang des Abflusses und einem Spitzenabfluss in der Größe von einem HQ100 (Abb. 5) am geplanten Standort der Wasserfassung. Der Geschiebeeintrag in den Stauraum 


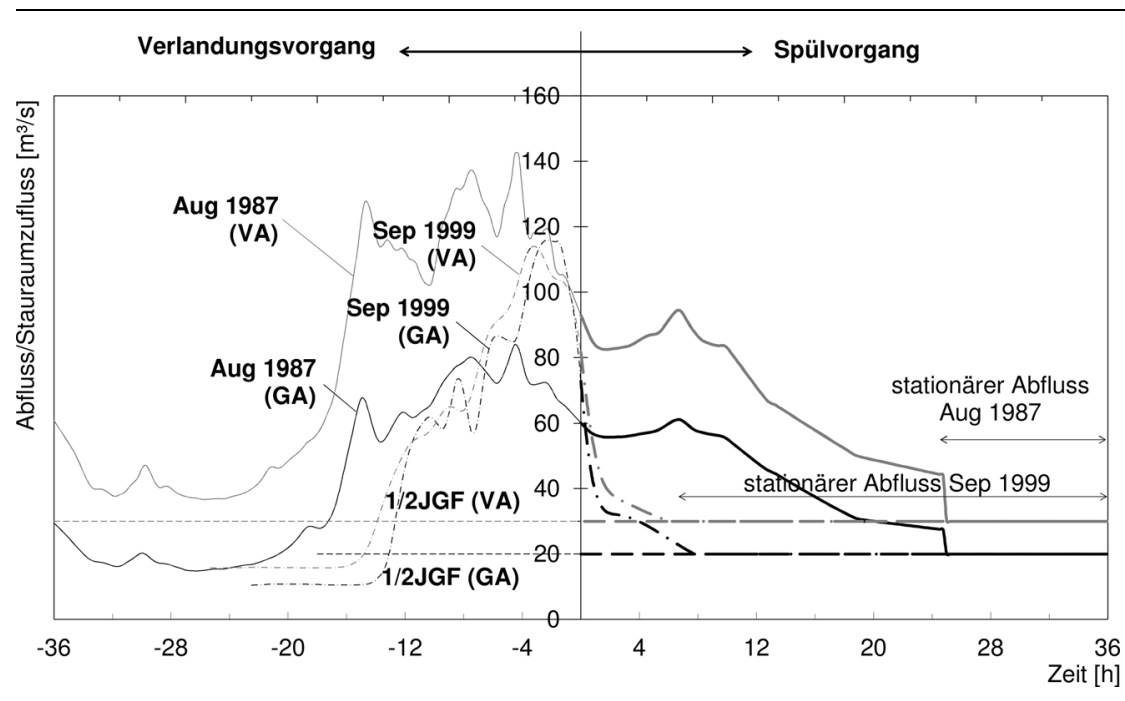

Abb. 5 Abfluss bzw. Hochwasserganglinien mit Definition des Spülbeginns für die Stauraumbelastungen 1/2 JGF, Aug 1987, Sep 1999 an der Gurgler Ache (GA) und an der Venter Ache (VA)

beträgt ca. $200 \%$ der Jahresgeschiebefracht $\left(16345 \mathrm{~m}^{3}\right)$.

\subsection{Spülmuster und Stellenwert des Freispiegelabflusses durch den Grundablass}

Die Spülung der halben Jahresgeschiebefracht an der Gurgler Ache basiert auf einem stationären Abfluss von $20 \mathrm{~m}^{3} / \mathrm{s}$. Die Frachten aus den Hochwasserereignissen wurden mit einem instationären Abfluss entsprechend des abfallenden Bereiches der Hochwasserganglinie gespült. Hier wurde zusätzlich manuell eingegriffen. $\mathrm{Ab}$ dem Zeitpunkt, $\mathrm{ab}$ dem keine Daten für die Hochwasserganglinie vorhanden waren, wurde vereinfachend ein Spülabfluss von $20 \mathrm{~m}^{3} / \mathrm{s}$ berücksichtigt (Abb. 5).

Die Erkenntnisse aus dem Modellversuch bestätigten erste Annahmen, dass bei Stauraumspülungen ein effektiver Geschiebetransport durch den Grundablass erst bei Erreichen eines Freispiegelabflusses auftritt. Damit spielt der Abstau (Vorgang vom Öffnen des Grundablasses bis zum Erreichen des Freispiegelabflusses) eine untergeordnete Rolle für die Bestimmung des Geschiebetransports durch den Grundablass. In den nachfolgenden Darstellungen der Ergebnisse wurde die Dauer des Abstauvorgangs nicht berücksichtigt. Die Spülleistungen beziehen sich damit nicht auf den Zeitpunkt der Öffnung des Grundablasses, sondern auf einen Nullpunkt, welcher den Beginn des Freispiegelabflusses definiert.

\subsection{Auswahl der Ergebnisse als Basis für die Kalibrierung des numerischen Modells}

\subsubsection{Verlandungsvorgang}

Die Verlandungsvorgänge zeigten, dass alle Stauraumbelastungen im Stauraum aufgenommen werden können (Abb. 6). Eine weiterführende Untersuchung hinsichtlich des Verlandungsverhaltens führte zu einem zusätzlichen Versuch mit der doppelten Stauraumbelastung eines Hochwassers August 1987 (550 \% der JGF). Selbst bei dieser äußert unwahrscheinlichen Belastung konnte keine Beeinträchtigung der Wasserfassung und der Betriebseinrichtungen zustände aus den Untersuchungen im physikalischen Modellversuch wurden mit vier Parametern beschrieben:

- Abstand der Verlandungsfront zum Grundablass/zur Mauer,

- relative Verlandungshöhe bezogen auf die Ausgangssohllage,

- absolute Höhe der Verlandungsfront und

- mittleres Gefälle des Verlandungskörpers.

Diese Werte bildeten die Vergleichsgrößen für die Kalibrierung der numerischen Simulationen der Verlandungsvorgänge. Ergänzend wurden für das Hochwasserereignis September 1999 Geschiebeproben aus dem Verlandungskörper im Modellversuch entnommen. Die ermittelten Kornverteilungen dieser Proben wurden mit den festgestellt werden. Die Verlandungs-
Kornverteilungen des Verlandungsmaterials in den numerischen Berechnungen verglichen (AB Wasserbau 2015a).

\subsubsection{Spülvorgang}

Die Vergleichsgrößen für die Stauraumspülung und die Spülung der Unterwasserstrecke sind die zeitbezogenen Spülfrachten (kumulativer Transport/ Spülleistungen) (dicke/rote Linien in Abb. 7). Zusätzlich wurden bei der Spülung der Geschiebefracht aus dem Hochwasserereignis September 1999 analog zum Verlandungsvorgang - Proben im Modellversuch entnommen, um diese mit den Ergebnissen in den numerischen Berechnungen vergleichen zu können.

Die Spülung einer halben Jahresgeschiebefracht mit einem stationären Zufluss von $20 \mathrm{~m}^{3} / \mathrm{s}$ ergibt eine notwendige Spüldauer von $4 \mathrm{~h}$. Im Bereich der Unterwasserstrecke können in diesen $4 \mathrm{~h}$ ca. $1000 \mathrm{~m}^{3}$ Feststoffvolumen gespült werden, womit es zunächst zu Auflandungen im Bereich der Unterwasserstrecke kommt, welche jedoch mit fortlaufender Spüldauer reduziert werden können.

Beim Hochwasser August 1987 kann die Ereignisfracht innerhalb von $7 \mathrm{~h}$ gespült werden. Aufgrund der damit auftretenden hohen Transportraten kommt es zu starken Auflandungen im Bereich der Unterwasserstrecke, welche aber wie bei der Spülung der halben Jahresgeschiebefracht wieder reduziert werden können. In $21 \mathrm{~h}$ können ca. $17500 \mathrm{~m}^{3}$ aus der unmittelbaren Unterwasserstrecke transportiert werden (Abb. 7).

Auch beim Hochwasser September 1999 zeigt die Stauraumspülung gute Erfolge (12 $600 \mathrm{~m}^{3}$ in $\left.7 \mathrm{~h}\right)$. Im Bereich der Unterwasserstrecke können in $9 \mathrm{~h}$ aber nur ca. $5000 \mathrm{~m}^{3}$ dieser Fracht transportiert werden. Der Hauptgrund liegt dabei in der schnellen Abnahme des Spülabflusses auf $20 \mathrm{~m}^{3} / \mathrm{s}$. Mit diesem Abfluss wird der Transport von grobem Geschiebematerial infolge eines Hochwassereintrages erschwert. Es verlängert sich damit die erforderliche Spüldauer für eine Reduktion der Auflandungen und den Transport aus der Unterwasserstrecke (Abb. 7). 

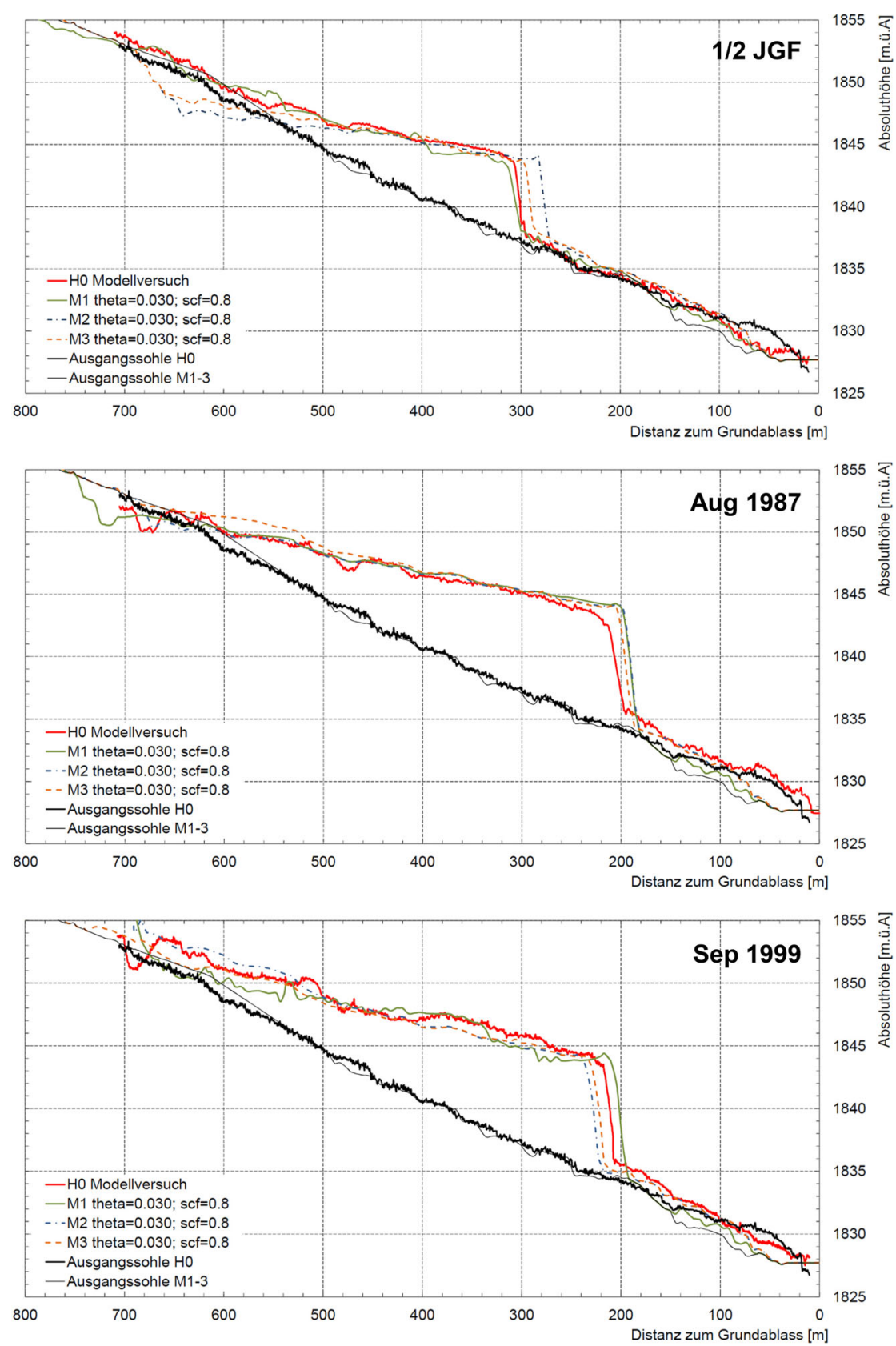

Abb. 6 ErgebnisseVerlandungsvorgang im physikalischen Modellversuch HO und den Simulationen M1, M2, M3 auf Basis von $\theta_{\text {crit }}=0.030$ und scf $=0.8$; oben: $1 / 2$ JGF; Mitte: Aug 1987; unten: Sep 1999

\section{Numerische Modellierung Wasserfassungen Gurgler Ache und Venter Ache (M1, M2, M3)}

\subsection{Allgemeines}

Für die Simulation der Verlandungsvorgänge reichen die Berechnungsnetze vom oberen Modellrand bis zum Auslauf aus der Unterwasserstrecke. Bei den Spülvorgängen bzw. bei Abfluss durch den Grundablass kommt es zu reicht von Grundablass bis zum Auslauf des Gesamtmodells (Abb. 8).

Die Bearbeitung mit zwei Teilmodellen entkoppelt den Spülvorgang im Stauraum von dem Spülvorgang in der Unterwasserstrecke und ist damit zunächst als Abweichung zur Spülung im Modellversuch zu sehen. Der Einfluss der Unterwasserstrecke auf die Stauraumspülung, insbesondere der Einfluss der Auflandungen im Nahbereich des Grundablasses, wird damit in den numerischen Berechnungen bewusst nicht berücksichtigt ( $\mathrm{AB}$ Wasserbau 2015a; Plörer et al. 2013a).

\subsection{Verwendete Software \\ 4.2.1 Strömungsberechnung}

Hydro_GS-2D ist eine Erweiterung der 2D-numerischen Strömungssimulationssoftware Hydro_AS-2D (Nujic 2009; Nujic 2015). Die Rechennetze werden mit einem linearen unstrukturierten Oberflächennetz aus Dreieck- und Viereckelementen erzeugt. Die Eigenschaften der Sohle und die Materialeigenschaften können dabei jedem Knoten bzw. jedem Element zugeordnet werden. Die Berechnung der hydraulischen Größen erfolgt durch numerische Lösung der vollständigen zweidimensionalen Flachwassergleichungen. Für die räumliche Diskretisierung wird die Finite-Volumen-Methode (FVM) und für die zeitliche Diskretisierung das RungeKutta-Verfahren 2. Ordnung (PrädiktorKorrektor-Methode) verwendet. Der Reibungsterm in den Gleichungen wird nach Darcy/Weissbach ermittelt.

\subsubsection{Transportberechnung/ Sohländerungen}

Die verwendete Version Hydro_GS-2D 3.14 erlaubt die Definition der Kornverteilungen des Geschiebes mit bis zu zwölf Korndurchmessern bzw. Kornklassen. In diesem Projekt wurden bezugnehmend auf die Arbeit von Umach (2014) die Kornverteilungen mit nur fünf Kornklassen definiert. Umach zeigt, dass der Mehrwert der Ergebnisse bei 2D-numerischen Berechnungen mit der Verwendung von mehr als fünf Kornklassen gering ist und die Rechenzeit durch diese Begrenzungen verringert werden kann. Für die Transportberechnung wird die von Hunziker erweiterte Formel von Meyer-Peter und Müller zur Modellierung von fraktioniertem Geschiebe verwendet (Hunziker 1995; 

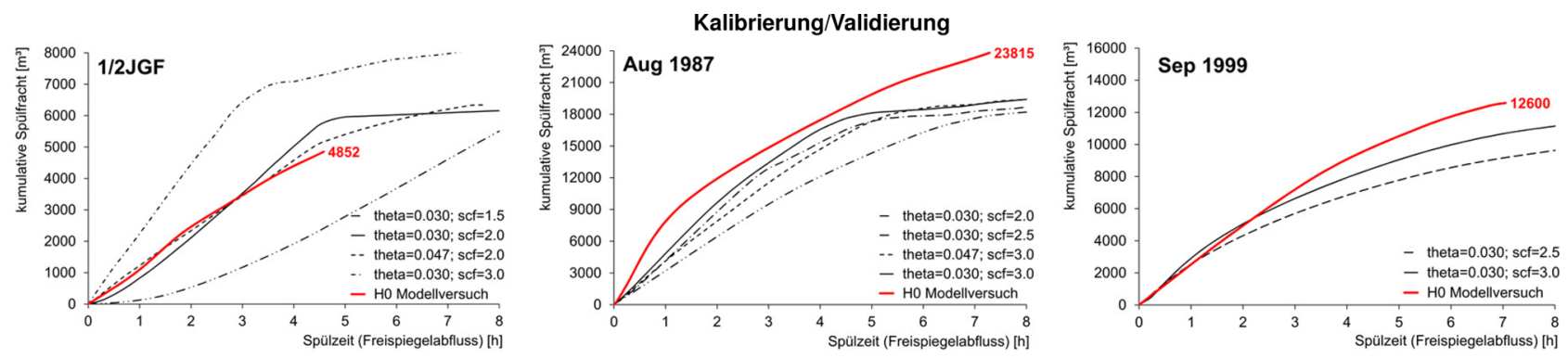

Ergebnisse Stauraumspülung
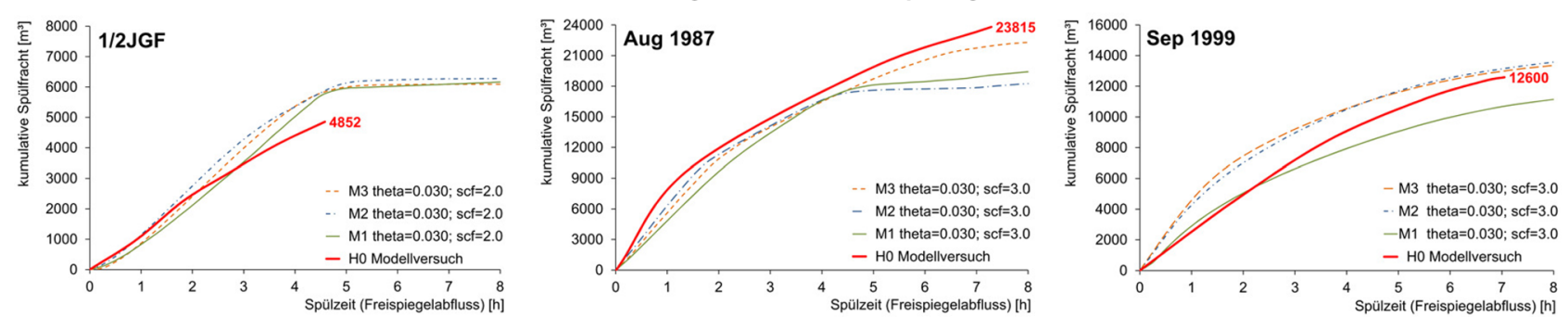

Ergebnisse Spülung Unterwasserstrecke
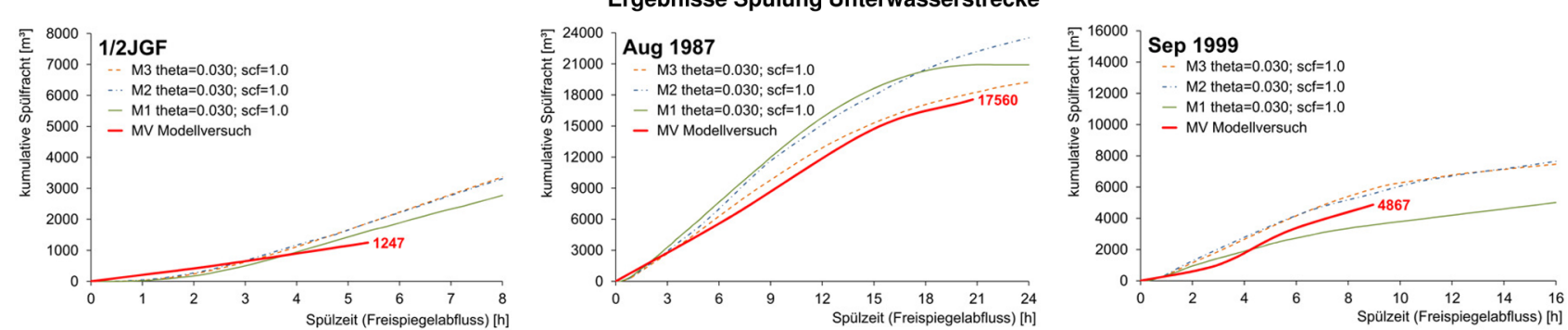

Abb. 7 Zeile 1: Ergebnisse der Kalibrierungsberechnungen für die Stauraumbelastung 1/2 JGF, HW 1987 und HW 1999; Zeile 2: Ergebnisse der Stauraumspülung für Modelltype M1, M2 und M3 auf Basis eines $\theta_{\text {crit }}=0.03$ und scf $=2.0(1 / 2 J G F)$ bzW. scf $=3.0$ (HW 1987, HW 1999); Zeile 3: Ergebnisse der Spülung der Unterwasserstrecke für Modelltyp M1, M2 und M3 auf Basis eines $\theta_{\text {crit }}=0.03$ und scf $=1.0(1 / 2$ JGF, HW 1987, HW 1999)

Hunziker et al. 2009). Die Berechnung des abgelagerten bzw. transportieren Geschiebes erfolgt mit den Exner-Gleichungen. Die Sohle wird mittels drei Schichten, der Austauschschicht, der Unterschicht und der Grundschicht dargestellt. Eine Einführung in die morphodynamische Modellierung und eine genaue Beschreibung der Modellkomponenten können im Handbuch für Hydro_GS-2D (Nujic 2015) und in den Arbeiten der angeführten Autoren gefunden werden.

\subsection{Beschreibung der Parameter für die Kalibrierung}

\subsubsection{Modellparameter}

Für die Kalibrierung des numerischen Modells kann zwischen den allgemeinen Modellparametern und Parametern für die Geschiebetransportformel unterschieden werden. Im Teilbereich „Modellparameter“ führt eine zeitlich und räumlich höher aufgelöste Diskretisierung zur Verbesserung der Darstellung von hoch instationären Prozessen. Der Nachteil einer feinen Diskretisierung liegt im klar erhöhten Rechenaufwand und damit in einer längeren Berechnungsdauer. Es sind folgende Maßnahmen möglich:

a) Verfeinerung des Berechnungsnetzes; insbesondere in Bereichen mit hoch-instationären Strömungsvorgängen.

b) Reduktion des Modellparameters $\mathbf{H}_{\text {min; }}$ dieser definiert die minimale Wassertiefe, die an einem Knoten vorherrschen muss, damit dieser zur Berechnung herangezogen wird. Eine Verkleinerung dieses Wertes ergibt eine Erhöhung der Rechenzeit, kann aber bei instationären Vorgängen zu besseren Ergebnissen führen.

c) Reduktion von Modellparameter $\mathbf{A}_{\text {min; }}$ definiert die minimale Elementgröße; das Residuum wird bei kleinen Elementen stärker gedämpft als bei großen. Bis zu dieser definierten Elementgröße kommt es zu keiner Dämpfung an den Elementen. Ein großer $\mathrm{A}_{\mathrm{min}}$-Wert kann das Erreichen eines stationären Zustandes verzögern.

d) Reduktion der Courant-FriedrichsLewy-Zahl (CFL-Zahl), eine indirekte Einflussnahme auf den diskreten Zeitschritt in der numerischen Berechnung:

$$
\mathrm{CFL}=\frac{\mathrm{u} \cdot \Delta \mathrm{t}}{\Delta \mathrm{x}}
$$

mit

u Geschwindigkeit

$\Delta \mathrm{t}$ diskrete Zeitschritt

$\Delta \mathrm{x}$ diskrete Ortschritt

e) Schubspannungsschwankungen: Werden Schubspannungsschwankungen nicht berücksichtigt, so wer- 

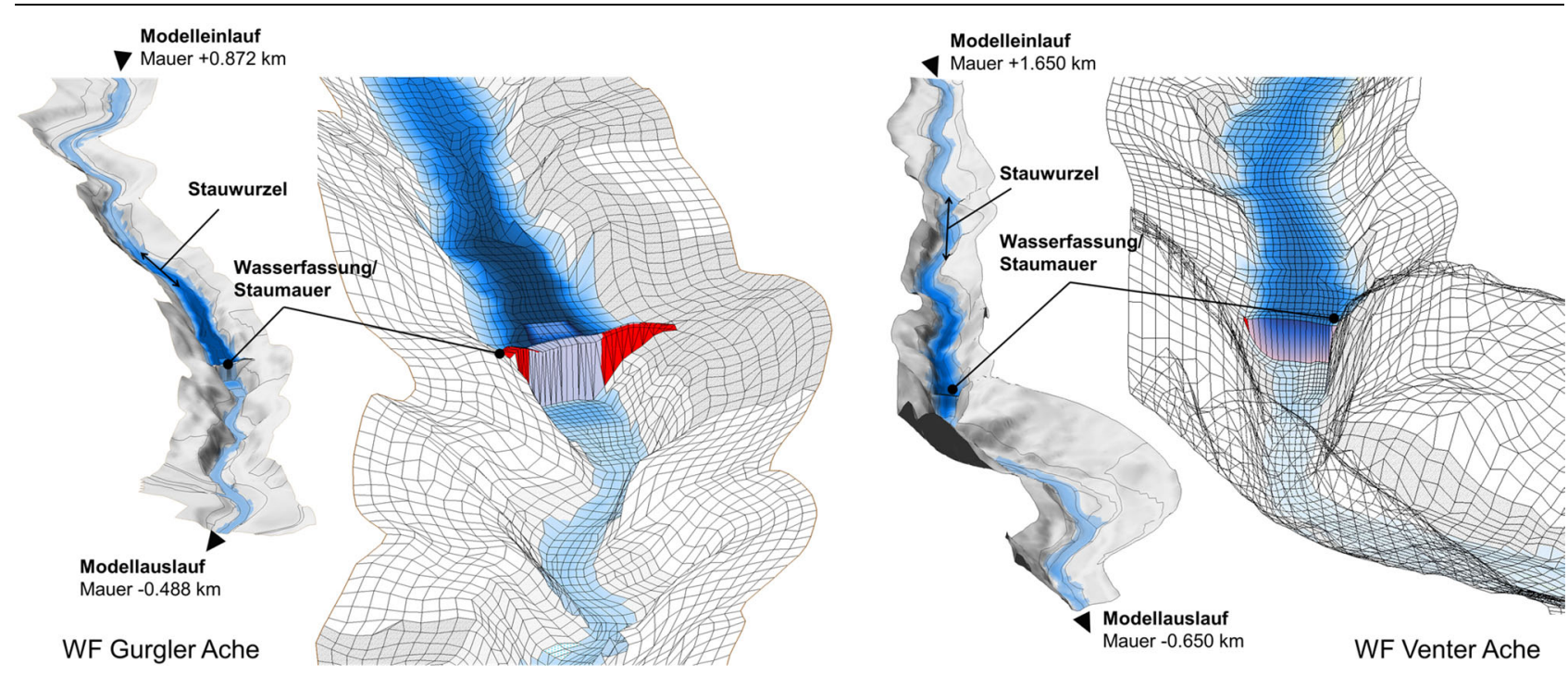

Abb. 8 Berechnungsnetze für die numerischen Simulationen; links: Gurgler Ache; rechts: Venter Ache

den einzelne Spannungsspitzen gedämpft und gemittelt. Das Einbeziehen der Schwankungen erhöht die Rechenzeit. Dennoch hat sich insbesondere bei den Spülvorgängen gezeigt, dass diese Spitzen den Transport begünstigen und zu höheren Spülleistungen führen.

f) Reduktion der Anpassungslänge $L_{a}$; die Anpassungslänge definiert das Verhältnis zwischen Transportkapazität und tatsächlichem Transport; geht die Anpassungslänge gegen Null so entspricht der Transport der Transportkapazität.

\subsubsection{Parameter für den Geschiebetransport}

Die Transportberechnungen für fraktioniertes Geschiebe basieren in Hydro_GS-2D auf der durch Hunziker (Hunziker 1995; Hunziker et al. 2009) adaptierten MPM-Formel (Meyer-Peter und Müller 1949):

$$
q_{b}=\mathbf{s c f} \cdot \sum F_{i} \cdot 8 \cdot\left(\varphi_{i} \cdot\left(\mu \cdot \theta-\theta_{c m s}\right)\right)^{(3 / 2)}
$$

mit

$$
\mu=\left(\frac{\boldsymbol{k s} \boldsymbol{t}}{\boldsymbol{k r}}\right)^{(3 / 2)} \cdot \frac{h \cdot J}{\left(\frac{\rho_{s}}{\rho_{w}}-1\right) \cdot d_{m s}}
$$

und

$$
\theta_{c m s}=\boldsymbol{\theta}_{\text {crit }} \cdot\left(\frac{d_{m s s}}{d_{m s}}\right)^{(1 / 3)}
$$

scf Skalierfaktor für den Geschiebetransport; Die MPM-Formel in ihrer ursprünglichen Form beinhaltete den Faktor 8 (Glg. 2). Weiterführende Untersuchungen erläutern, dass dieser Faktor eher geringer ist und bei ca. 5 liegt. Dieser Wert ist auch der Startwert in Hydro_GS-2D und wird indirekt durch einen scf-Wert von $0.625(=5 / 8)$ erreicht

\section{$\mathrm{F}_{\mathrm{i}} \quad$ Fraktion $\mathrm{i}$}

$\varphi_{i} \quad$ Ausgleichsfunktion

$\mu \quad$ Faktor aus dem Verhältnis Gesamtrauheit zu Kornrauheit und Shields-Parameter

$\theta_{\mathrm{cms}}$ kritische dimensionslose Schubspannung einer Mischung, bezogen auf den mittleren Korndurchmesser der Austauschschicht

$\theta_{\text {crit }}$ kritische dimensionslose Schubspannung für Einheitskorn

$\mathrm{d}_{\mathrm{mss}}$ charakteristischer Korndurchmesser der Unterschicht

$\mathrm{d}_{\mathrm{ms}}$ charakteristischer Korndurchmesser der Ausgleichsschicht

Die ersten Berechnungen mit den Default-Parametern (Grundeinstellungen des Programms) zeigten sehr geringe Spülleistungen, teilweise waren auch numerische Instabilitäten im Bereich des Grundablasses zu beobachten. Mit den oben angeführten Maßnahmen für die Modellparameter alleine wurden die Ergebnisse aus dem Modellversuch nicht erreicht. Die Spülleistungen im Modellversuch übertrafen die Spülleistungen in der Numerik um das Zweibis Dreifache. Damit wurde eine Ka- librierung der Transportvorgänge mit Einflussnahme auf die Geschiebetransportformel notwendig.

Aus der Formelstruktur ist ersichtlich, dass u. a. der scf-Wert, der Faktor $\mu$ und die dimensionslose kritische Sohlschubspannung $\theta_{\text {crit }}$ einen Einfluss auf den Transport haben. Im Zuge der Kalibrierung und der Variantenberechnung zeigte sich, dass die Simulationen auf eine Veränderung dieser Parameter am stärksten reagieren. Der scf-Wert beeinflusst den Transport linear, d. h. eine Verdoppelung dieses Werts führt in Abhängigkeit von der Transportkapazität bis zu einer Verdoppelung des Transports. Einen wichtigen Einfluss auf das gesamte Transportverhalten hat die dimensionslose kritische Sohlschubspannung $\theta_{\text {crit. }}$ Diese ist entsprechend der Untersuchungen nach MPM mit 0.047 angesetzt, kann aber in Hydro_GS-2D manuell definiert werden. Der Faktor $\mu$ wird durch das Verhältnis von Gesamtrauheit zur Kornrauheit im Gerinne bestimmt. Werden beide Größen ident angenommen, so ergibt sich keine Verkleinerung des Transports durch den Faktor $\mu$,da „, $\left(\mathrm{k}_{\mathrm{st}} / \mathrm{k}_{\mathrm{r}}\right)^{\wedge}(3 / 2)^{\prime \prime}$ in Gleichung 3 eins ergibt.

\subsection{Kalibrierung und Validierung}

\subsubsection{Stauraumverlandung}

Die ersten Berechnungen für den Verlandungsvorgang infolge einer Stauraumbelastung mit einer halben Jahresgeschiebefracht basieren auf einer dimensionslosen kritischen Sohlschub- 
spannung von 0.047 und einem scfWert von 0.625 (entspricht 5/8). Dieser scf-Wert definiert den Einfluss von Formrauheiten, welcher zu einer Verkleinerung des Geschiebetransports führt. Die Resultate dieser Berechnungen zeigten bereits eine große Übereinstimmung mit den Ergebnissen aus dem Modellversuch. Es kam noch vereinzelt zu starken Auflandungen im Bereich der Zulaufstrecke, welche so nicht im Modellversuch beobachtet wurden. Eine geringfügige Erhöhung des scfWertes auf 0.8 bzw. die Reduktion des Einflusses von Sohlformen und die Verringerung von $\theta_{\text {crit }}$ führten schließlich $\mathrm{zu}$ den besten Resultaten und zeigten eine sehr gute Übereinstimmung des Verlandungskörpers nach Modelltyp M1 mit jenem aus dem physikalischen Modellversuch H0 (Abb. 6). Diese Einstellungen wurden für die Modellierung der Verlandung infolge der Hochwasserereignisse August 1987 und September 1999 übernommen und es konnten auch hier sehr gute Ergebnisse erreicht werden. Abb. 6 zeigt den Vergleich der Verlandungskörper im Modelltyp M1 und im Modellversuch H0 für das Hochwasserereignis August 1987 und die Ergebnisse der Verlandungsvorganges für das Hochwasserereignis September 1999.

\subsubsection{Stauraumspülung}

Im Gegensatz zur Stauraumverlandung erforderte die Spülung des Stauraums einen längeren Kalibrierungsprozess. Mit der Reduktion von $\theta_{\text {crit }}$ und einer Erhöhung des scf-Wertes auf den laut Literatur maximalen Wert von 1.0 konnte der Transport erhöht werden, jedoch waren die Spülfrachten deutlich geringer als im Modellversuch. Daraufhin wurden in Anlehnung an Abschnitt 4.3 Maßnahmen gesetzt, um den Transport weiter $\mathrm{zu}$ verbessern. Insbesondere die Veränderung der Anpassungslänge $\mathrm{L}_{\mathrm{a}}$ resultierte in einem starken Anstieg des Geschiebetransportes. Dennoch konnten immer noch nicht die Referenzwerte aus dem Modellversuch erreicht werden. Als letzter Schritt wurde mit einem scf-Wert größer als eins versucht, den Transport zu verstärken. Mit $\theta_{\text {crit }}=0.030$ und $\mathrm{scf}=1.5$ liegt der Transport noch deutlich unter jenem im Modellversuch (Abb. 7). Eine zusätzliche Steigerung auf 2.0 führte zu guten Resultaten. Ein Test mit scf $=3.0$ zeigte einen zu großen Geschiebetransport. Die Parametereinstellungen $\theta_{\text {crit }}=0.030$ und $\operatorname{scf}=2.0$ wurden daraufhin für die Bestimmung der Stauraumspülung mit den Hochwasserbelastungen August 1987 und September 1999 verwendet. Hier zeigte sich, dass mit einem scf $=2.0$ nicht der gewünschte Transport erreicht wird. Damit wurde der scf-Wert weiter erhöht, zunächst auf 2.5, und schließlich auf 3.0. Für beide Hochwasserereignisse konnte mit scf $=3.0$ ein Transport ermittelt werden, welcher nah an jenem im Modellversuch liegt (Abb. 7). Beim Hochwasser August 1987 kommt es in den numerischen Berechnungen $\mathrm{zu}$ einem verminderten Transport am Ende des Spülvorganges, welcher so im Modellversuch nicht beobachtet werden konnte. Der Versuch einer weiteren Anpassung an die Ergebnisse aus dem Modellversuch konnte auch mit Simulationen basierend auf einen scf-Wert von 4.0 nicht erreicht werden. Insbesondere im Bereich des Grundablasses traten numerische Instabilitäten auf.

\subsubsection{Spülung Unterwasserstrecke}

Im Bereich der Unterwasserstrecke liegen die hydraulischen Bedingungen näher an gewohnten Fließzuständen in offenen, natürlichen Gerinnen als im Bereich des Stauraums. Im Zuge der Kalibrierung konnte bei der Spülung der halben Jahresgeschiebefracht mit $\theta_{\text {crit }}=0.030$ und einem scf-Wert von 1.0 eine gute Übereinstimmung zwischen Modellversuch und Numerik erreicht werden. Eine Übertragung dieser Stellgrößen zu den Berechnungen der Hochwasserereignisse August 1987 und September 1999 führte auch dort zu Übereinstimmungen. Zusätzlich zeigt die Auswertung der Auflandungs- und Erosionsmuster während der Spülung die gleichen Eigenschaften wie im Modellversuch. Die Auflandungen infolge der hohen Spülfrachten werden mit fortlaufendem Spülvorgang wieder reduziert. Insbesondere bei einer Belastung mit einer halben jährlichen Geschiebefracht können die Auflandungen nach $12 \mathrm{~h}$ wieder mobilisiert werden (Abb. 7, Abb. 9). Beim Hochwasserereignis August 1987 kann ein Großteil des Geschiebes aus dem Stauraum innerhalb von $24 \mathrm{~h}$ aus der unmittelbaren Unterwasserstrecke transportiert werden. $\mathrm{Zu}$ stärkeren Ablagerungen kommt es beim Hochwasserereignis September 1999, wo innerhalb $16 \mathrm{~h}$ nur etwa die Hälfte der Ereignisfracht transportiert werden kann (Abb. 7). Hier sind für einen weiteren Abbau der Ab- lagerungen in der Unterwasserstrecke eine längere Spüldauer und ein höherer Spülabfluss notwendig.

\section{5 Übertragungen der} Modellparameter von Modelltyp M1 auf M2 und M3

Die Modellparameter der kalibrierten Modelle M1 wurden schließlich auf den Modelltyp M2 übertragen. Neben einer geometrischen Skalierung der Berechnungsnetze umfasste dieser Vorgang auch die Skalierung der Kornverteilung des Geschiebes mit dem Maßstabsfaktor 30. Auf Basis dieser Einstellungen wurden die gleichen Simulationen wie beim Modelltyp M1 durchgeführt. Der Übertragungsschritt hin zum Modelltyp M3 beinhaltete nun noch die Berücksichtigung der feinsten Anteile in den Kornverteilungen. Auch hier wurden sowohl die Verlandungsvorgänge, die Stauraumspülungen und die Spülungen der Unterwasserstrecke für die Belastung aus einer halben Jahresgeschiebefracht und mit den Hochwasserereignissen August 1987 und September 1999 untersucht.

Die Modellparameter der Simulationen "Gurgler Ache“ Modelltyp M3 für die drei Teilbereiche Stauraumverlandung, Stauraumspülung und Spülung der Unterwasserstrecke wurden in einem letzten Schritt zur numerischen Simulation für die „Venter Ache“ Modelltyp M3 übertragen. Die Simulationen umfassen hier das gleiche Modellierungsprogramm wie bei der Gurgler Ache unter Berücksichtigung eines höheren stationären Abflusses für den Spülvorgang im Stauraum und in der Unterwasserstrecke $\left(\mathrm{Q}=30 \mathrm{~m}^{3} / \mathrm{s}\right)$ (Abb. 5). Bedingt durch die Nähe der beiden Standorte der Wasserfassungen und der Rahmenbedingungen für die hydrologische Modellierung zeigen sich qualitativ ähnliche Abflussganglinien für die Hochwasserereignisse wie an der Gurgler Ache. Der Spitzenabfluss beträgt für das Hochwasser August 1987 $142 \mathrm{~m}^{3} / \mathrm{s}$ und für das Hochwasser September $1999114 \mathrm{~m}^{3} / \mathrm{s}$. Die halbe Jahresgeschiebefracht wurde am geplanten Standort der Wasserfassung Venter Ache mit $8960 \mathrm{~m}^{3}$ berechnet. Die Ereignisfrachten betragen $60572 \mathrm{~m}^{3}$ für das Hochwasser August 1987 und 21584 m $^{3}$ für das Hochwasser September 1999 (Tab. 1). 


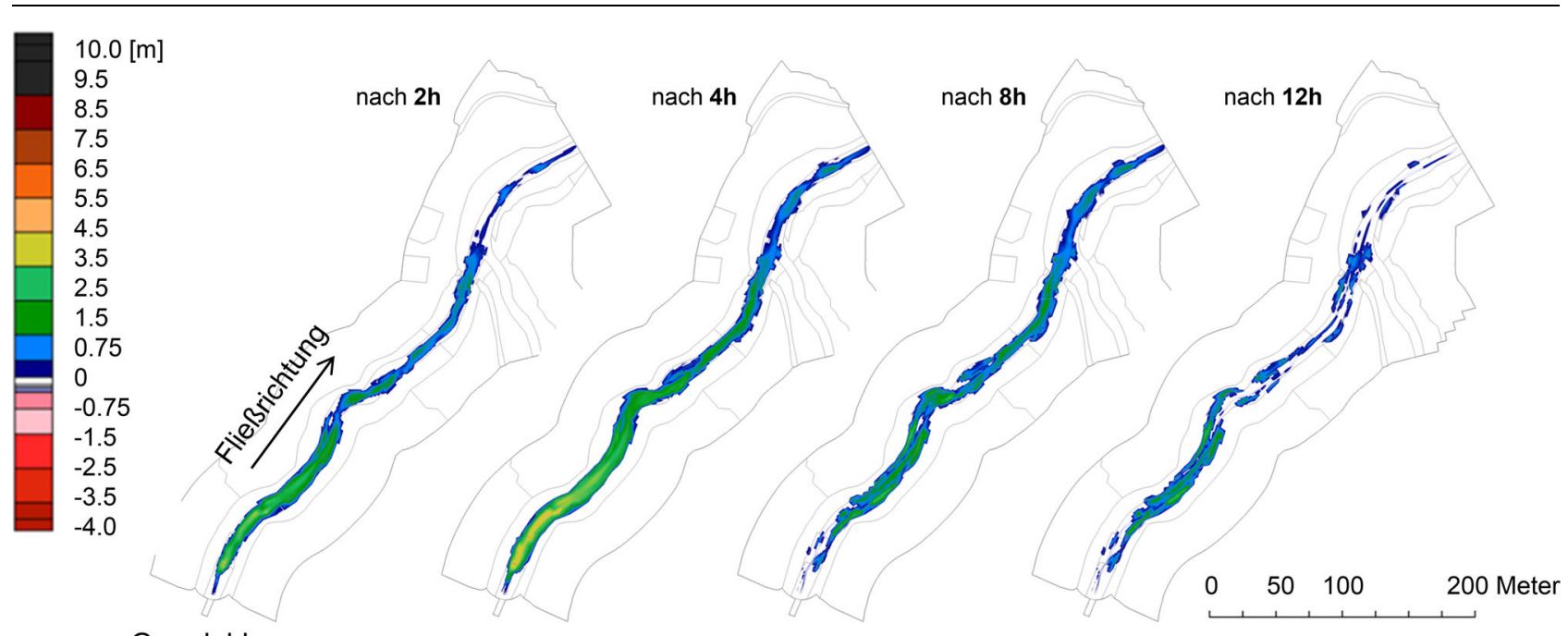

Grundablass

Abb. 9 Entwicklung der Geschiebeablagerungen in der Unterwasserstrecke während der Stauraumspülung der halben Jahresgeschiebefracht (1/2 JGF) an der Wasserfassung Gurgler Ache

\subsection{Ergebnisse}

Abb. 7 zeigt die Gegenüberstellungen der Resultate aus den numerischen Berechnungen mit den Modelltypen M1, M2 und M3 mit jenem aus dem Modellversuch, H0. In den Ergebnissen sind leichte Abweichungen zwischen den Modelltypen erkennbar. Während bei einer halben Jahresgeschiebefracht und beim Hochwasserereignis August 1987 nur geringe Unterschiede auftreten, so erkennt man eine größere Abweichung zwischen den Berechnungen im Modelltyp M1 zu M2 und M3 beim Hochwasserereignis September 1999. Mit einer Gesamtbeurteilung der Resultate lässt sich keine generelle Aussage über die Gründe dieser Ergebnisse treffen. Betrachtet man die Resultate mit einer halben Jahresgeschiebefracht und beim Hochwasserereignis August 1987, so zeigt sich, dass es weder durch die Veränderung des Modellmaßstabs (Modelltyp M1 <> Modelltyp M2, M3), noch aufgrund der Berücksichtigung der Feinanteile (Modelltyp M1, M2 <> Modelltyp M3) zu wesentlichen Änderungen des Transportes kommt. Eine Erklärung kann im starken Abfall des Spülabflusses beim Hochwasserereignis September 1999 liegen, welcher sich in den numerischen Simulationen im Modellmaßstab möglicherweise stärker auswirkt.

\subsection{Bewertung der Parameterwahl}

Die Verlandungsprozesse sind aufgrund der hydraulischen und mor- phologischen Randbedingungen (langsame Änderung der Verlandung, kleine Energielinien- und Sohlgefälle, geringe Fließgeschwindigkeiten) mit der 2D-numerischen morphodynamischen Software Hydro_GS-2D sehr gut zu simulieren. Allerdings ist die angenommene dimensionslose kritische Sohlschubspannung $\theta_{\text {crit }}$ mit 0.030 sehr klein. Die hoch instationären Erosionsund Auflandungsprozesse während der Stauraumspülung (schnelle und große Sohlveränderungen, große Energieliniengefälle und Fließgeschwindigkeiten) führen in der Modellierung zu Problemen, die nicht vollständig gelöst werden konnten. Bei einer zweidimensionalen hydrodynamischen Berechnung sind Vereinfachungen notwendig, die Auswirkungen auf die Berechnungen haben und damit Abweichungen zu den Abläufen in der Natur darstellen. Um näher an die Ergebnisse des physikalischen Modellversuchs zu kommen, sind Parametereinstellungen in den Simulationen zu treffen, die von Literaturwerten abweichen.

Im Bereich der Unterwasserstrecke konnten die Ergebnisse aus dem Modellversuch mit einem scf-Wert von 1.0 erreicht werden, welcher die nach Literaturwerten obere Grenze darstellt und den Einfluss von Formrauheiten nicht berücksichtigt. Auch hier wurde eine kleine dimensionslose kritische Sohlschubspannung $\left(\theta_{\text {crit }}=0.030\right)$ verwendet.

Trotz der angeführten Parameterwahl zeigt die Gesamtbetrachtung der Ergebnisse, dass ein Modellkonzept, das die Übertragung der Modellparameter eines kalibrierten numerischen Modell Gurgler Ache im Modellmaßstab (M1) auf ein numerisches Modell in Naturmaßstab (M2/M3) mit anschließender Übertragung auf das numerische Modell Venter Ache vorsieht, seine Berechtigung findet. Auch für den Verlandungsprozess an der Venter Ache konnten plausible Resultate erzielt werden. Bei der Simulation der Spülung des Stauraums konnten ähnliche Abhängigkeiten der Spülerfolge von einzelnen Modellparametern $\left(\theta_{\text {crit }}\right.$, scfWert) beobachtet werden wie bei der Gurgler Ache (AB Wasserbau 2015a).

\section{Naturmessungen und numerische Modellierung Wasserfassung Taschachbach (N1)}

\subsection{Kurzbeschreibung Wasserfassung Taschachbach}

Die Wasserfassung Taschachbach ist die größte Wasserfassung des Kraftwerks Kaunertal und liegt im Pitztal/ Tirol auf $1800 \mathrm{~m}$. ü. A. (Stauziel). Die Fassung befindet sich an einer kleinen Bogenstaumauer, welche überströmbar ausgeführt ist und damit zur Entlastung herangezogen werden kann. Die Fassung wurde als Frontentnahme am Sperrenbauwerk konzipiert und kann bis zu $12 \mathrm{~m}^{3} / \mathrm{s}$ ausleiten. Die Entnahmeleitung führt durch die Bogenmauer zu den beiden Entsandern am orografisch linken Ufer. Durch den Überleitungstollen wird das Wasser dem Speicher 

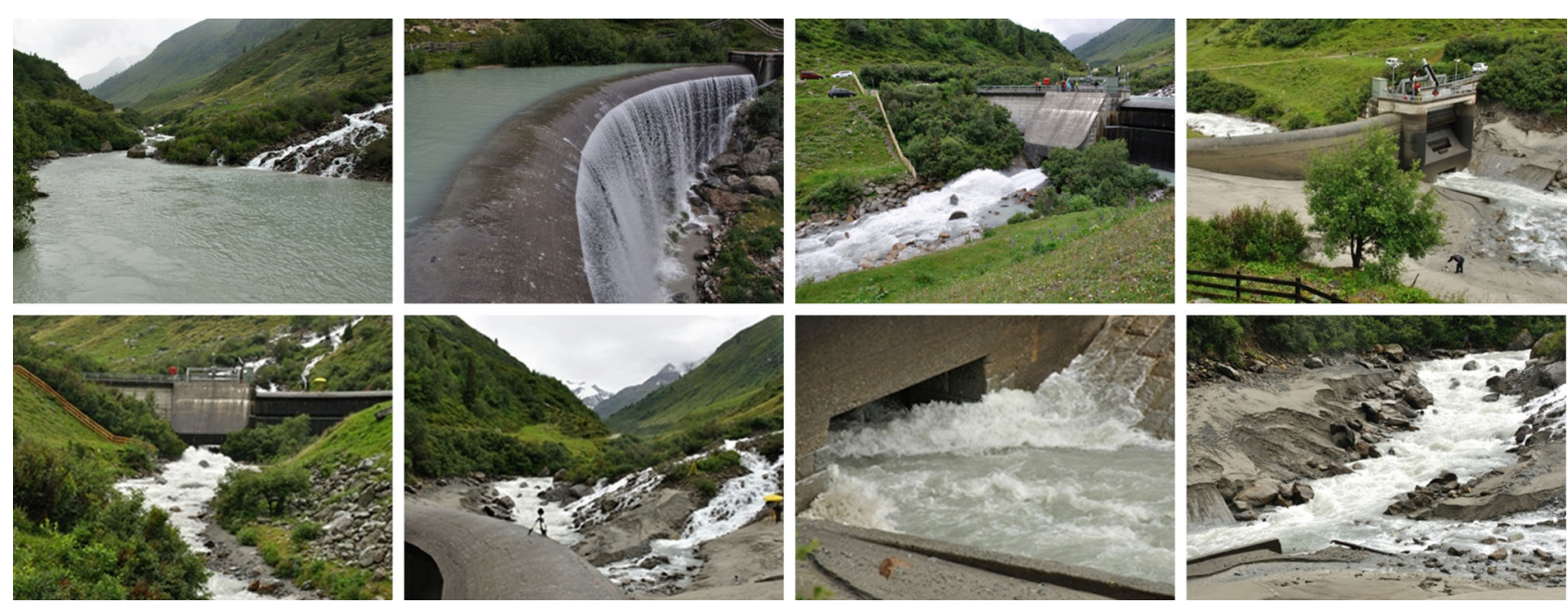

Abb. 10 Stauraumspülung Wasserfassung Taschachbach am 24.07.2014

Gepatsch im Kaunertal zugeführt. Das gesamte Einzugsgebiet der Fassung beträgt ca. $60.6 \mathrm{~km}^{2}$ und ist etwa zu einem Fünftel vergletschert. Aufgrund des vorhandenen Moränenmaterials gibt es ein großes Potenzial an Sedimenten, welches in den Stauraum transportiert werden kann. Aus diesem Grund wird der Stauraum mit einer hydraulischen Spülung durch den Grundablass im Mittel zwei- bis dreimal jährlich gespült. Während der Spülung wird kein Wasser eingezogen, womit der volle Zufluss für die Spülung zu Verfügung steht (AB Wasserbau 2015b). Die langjährigen Aufzeichnungen zeigen, dass bei den Regelspülungen sehr gute Spülerfolge erreicht werden (Tschada und Hofer 1990).

\subsection{Beschreibung der Naturmessungen}

Am 24.07.2014 führte die TIWAG eine Spülung des Stauraums der Wasserfassung Taschachbach durch (Abb. 10). Im Zuge dieser Regelspülung wurde ein umfangreiches Messprogramm abgewickelt (AB Wasserbau 2015b):

- Vermessung des Stauraums vor und nach der Spülung: Vor der Spülung wurde der Stauraum mit Lotmessungen aus einem Boot vermessen. Nach der Spülung bzw. bei freiem Durchfluss wurde der Stauraum mittels Laser aufgenommen. Damit wurde der Verlandungszustand abgeleitet und in das numerische Modell übertragen. Aufbauend auf den plausiblen Ergebnissen der Verlandungsmodellierung an der Gurgler und Venter Ache wurde auf eine zusätzliche numerische Modellierung der Stauraumverlandung verzichtet. Mit dem Vergleich der Stauraumverlandung vor und nach der Spülung konnte das gespülte Feststoffvolumen (Referenzvolumen) für die numerische Simulation mit $440 \mathrm{~m}^{3}$ bestimmt werden.

- Aufnahme der hydrologischen Bedingungen: Für die Spülung wurde das zeitliche Intervall der Aufnahme des Abflusses an einem Pegel in der Unterwasserstrecke auf 1 Minute reduziert. Mit dieser Abflussganglinie konnten der Zufluss zur Fassung und der Spülabfluss rekonstruiert werden.

- Messungen an 7 Querprofilen in der Unterwasserstrecke umfassten $u$. a. sogenannte Trübemessungen zur Bestimmung des Schwebstoffgehalts.

- Bestimmung der Kornverteilungen des Verlandungsmaterials: Während der Spülung wurden im Stauraum Proben des Verlandungskörpers entnommen, die im Labor des Arbeitsbereichs Wasserbau gesiebt und ausgewertet wurden. Damit ergeben sich wichtige Informationen über die Kornzusammensetzung, welche schließlich auch in das numerische Modell implementiert werden.

- Vermessung der Unterwasserstrecke und Bestimmung des Sohlmaterials in der Unterwasserstrecke im Nahbereich der Grundablassöffnung mit Linienzahlanalysen. Diese Informationen von der Ausbildung der Deckschicht und der Unterschicht sind eine wichtige Eingangsgröße für weitere geplante numerischen Simulationen.
- Begleitende Foto- und Videodokumentation.

Da sich die Daten der Sohllagen im Stauraum auf den Anfangs- und Endzustand im Stauraum beschränken, kann ein genauer zeitlicher Verlauf der Stauraumspülung nicht bestimmt werden und es sind vereinfachte Annahmen zu treffen. Der sehr beschränkte Sedimenttransport vor Erreichen des Freispiegelabflusses kann durch die Trübemessungen im Unterlauf und durch visuelle Beobachtungen bestätigt werden. Der eigentliche Geschiebetransport beginnt erst nach ca. $1 \mathrm{~h}$ nach Öffnung des Grundablasses mit Freispiegelabfluss. Aus diesen Erkenntnissen leitet sich eine „Spülkurve“ bzw. ein Bereich nach Abb. 11 ab, der durch die minimale Spüldauer $t_{f, \min }$ und die maximale Spüldauer $t_{f, \max }$ bestimmt ist. Laut visuellen Beobachtungen und Ableitungen aus den Trübemessungen wurde das Referenzvolumen von $440 \mathrm{~m}^{3}$ innerhalb dieses Zeitraums transportiert.

\subsection{Zielsetzung der numerischen Untersuchung N1}

Die numerische Nachrechnung der Stauraumspülung der Wasserfassung Taschachbach ermöglicht eine Verifizierung modellrelevanter Parameter, welche für die Simulation der Spülungen an den geplanten Wasserfassungen Gurgler Ache und Venter Ache verwendet wurden. Die wichtigsten Modellparameter und Einstellungen sind die dimensionslose kritische Sohlschubspannung $\theta_{\text {crit, }}$ der scf-Wert für die Geschiebetransportformel und die zeit- 


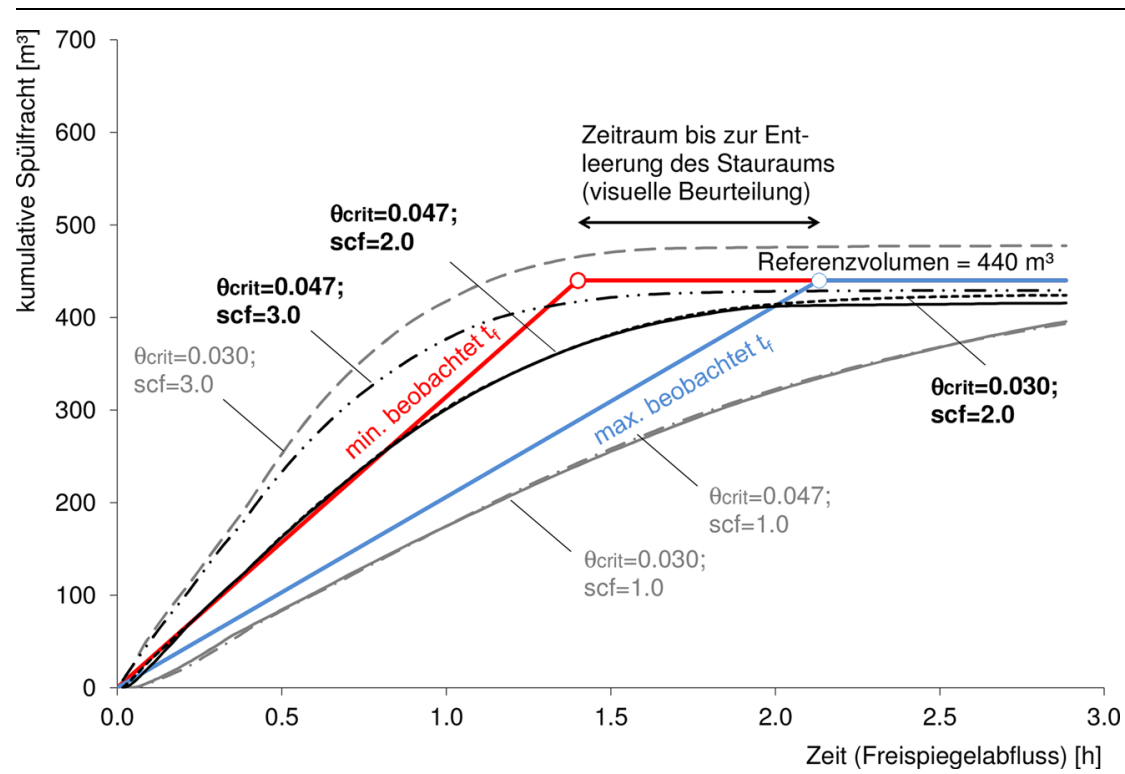

Abb. 11 Ergebnisse der numerischen Simulation der Stauraumspülung an der Wasserfassung Taschach; Spülleistungen in Abhängigkeit von $\theta_{\text {crit }}$ und dem scf-Wert

liche und räumliche Diskretisierung des numerischen Modells.

\subsection{Wahl der Modellparameter für die numerische Modellierung der Stauraumspülung Taschachbach}

Die Modellparameter wurden aufbauend auf den vorangegangenen Untersuchungen an den geplanten Wasserfassungen gewählt. Für eine Variantenuntersuchung wurden sowohl der scf-Wert als auch die dimensionslose kritische Sohlschubspannung $\theta_{\text {crit }}$ und die Anpassungslänge $\mathrm{L}_{\mathrm{a}}$ variiert. Erste Berechnungen mit hohen Anpassungslängen führten $\mathrm{zu}$ sehr geringen Spülleistungen. Damit wurde eine kleine und konstante Anpassungslänge für alle weiteren Berechnungen verwendet. Die Detailuntersuchung reduzierte sich damit auf sechs Varianten mit drei verschiedenen scf-Werten $(1.0 ; 2.0 ; 3.0)$ und zwei verschiedenen dimensionslosen kritischen Sohlschubspannungen $\theta_{\text {crit }}(0.030 ; 0.047)$.

\subsection{Ergebnisse}

Die Berechnungsvarianten mit scf $=1.0$ zeigen am Ende der Spülung eine kumulative Spülfracht von ca. $400 \mathrm{~m}^{3}$, womit $91 \%$ des Referenzvolumens gespült werden können. Trotzdem sind hier die Ergebnisse nicht zufriedenstellend, da sich klare Abweichungen zu den hohen Spülraten am Beginn der Spülung ergeben und damit die Dauer für die Entleerung des Stauraums nicht mit den Naturbeobachtungen übereinstimmt (Abb. 11). Die Varianten mit $\theta_{\text {crit }}$ $=0.030$ und einem scf-Wert von 2.0 und 3.0 und jene mit $\theta_{\text {crit }}=0.047$ und einem scf-Wert von 2.0 zeigen eine kumulative Spülfracht zwischen $96 \%$ und $99 \%$ des Referenzvolumens $\left(416 \mathrm{~m}^{3}\right.$ bis $\left.430 \mathrm{~m}^{3}\right)$. Bei der Variante $\theta_{\text {crit }}=0.030$ und scf $=3.0$ fällt der Transport zu groß aus. Hier werden bereits $110 \%\left(478 \mathrm{~m}^{3}\right)$ gespült.

Eine zusätzliche Bestimmung des zeitlichen Verlaufs der Spülung ist bedingt über die Interpretation der Schwebstofffrachten im Unterwasser möglich. Auch wenn eine Gegenüberstellung von Geschiebetransport mit Schwebstofftransport mit Vorbehalt zu verwenden ist, so lässt sich zumindest ein qualitativer Vergleich hinsichtlich des zeitlichen Verlaufes der Spülung des Verlandungsmaterials darstellen. Vor Beginn des Freispiegelabflusses ist nur eine geringe Konzentration zu erkennen. Der Maximalwert wird laut den Naturmessungen nach ca. 10 Minuten nach Beginn des Freispiegelabflusses erreicht (48 $000 \mathrm{mg} / \mathrm{l}$ ) (Abb. 12). Rechnet man nun die Feststofffrachten am Grundablass von $[\mathrm{kg} / \mathrm{s}]$ vereinfachend in eine Konzentration in [mg/l] um, so zeigt sich ein Vergleich der Feststoffkonzentrationen. Bei den vier Varianten mit einem scf-Wert von 2.0 bzw. 3.0 treten die Maxima auch nach ca. 10 Minuten Freispiegelabfluss auf. Betrachtet man die Spülraten, so zeigt sich, dass die Varianten mit einem $\theta_{\text {crit }}$ von 0.030 bzw. 0.047 besser mit der Trübemessung übereinstimmen. $\mathrm{Da}$ Trübemessungen nach $1 \mathrm{~h}$ nach Beginn des Freispiegelabflusses nicht vorhanden sind, kann keine Aussage getroffen werden, inwieweit die Varianten mit $\theta_{\text {crit }}=0.030 / \mathrm{scf}=2.0$ und mit $\theta_{\text {crit }}=0.047 / \mathrm{scf}=2.0$ in Folge mit den Messungen übereinstimmen.

Mit einer Gesamtbeurteilung der Ergebnisse ist davon auszugehen, dass die numerischen Simulationen mit Modellparameter von $\theta_{\text {crit }}$ Zwischen 0.030 und 0.047 und einem scf-Wert von 2.0 die Spülung sehr gut abbilden. Obwohl die Charakteristiken der Spülungen auch bei den übrigen Berechnungsvarianten zu plausiblen Abläufen führen, ist festzuhalten, dass mit $\theta_{\text {crit }}=0.030$ und einem scf-Wert von 3.0 der Transport überschätzt wird und die Spülraten bei den Varianten mit einem scf-Wert von $1.0 \mathrm{zu}$ gering sind.

\section{Zusammenfassende Interpretation des Modellkonzeptes}

Bei der numerischen Modellierung von Verlandungs- und Spülvorgängen an den Wasserfassungen Gurgler Ache und Venter Ache werden insbesondere bei der Stauraumspülung komplexe hydraulische und morphologische Vorgänge abgebildet. Durch das Absenken des Wasserspiegels und die rasche Veränderung des Verlandungskörpers treten hoch instationäre Prozesse auf. Die Simulation dieser Vorgänge mit dem gewählten 2D-numerischen geschiebehydraulischem Modell erfordert die Verwendung von Modellparametern, welche von gängigen Literaturwerten abweichen und damit auch zu neuen Fragestellungen führen.

Obwohl den Simulationen Ergebnisse aus einem Modellversuch im Maßstab 1:30 zugrunde liegen und damit sehr belastbare Vergleichsgrößen vorhanden sind, bietet die numerische Nachrechnung einer realen Spülung eine zusätzliche sinnvolle Möglichkeit, Eingangsgrößen in bisherigen Simulationen $\mathrm{zu}$ bewerten und Resultate $\mathrm{zu}$ verifizieren. Eine Beurteilung der Modellierungen auf Basis aller durchgeführten Simulationen zeigt, dass die Parameterwahl vom untersuchten Teilprozess abhängig ist:

- Mit den 2D-numerischen Simulationen können die Verlandungsprozesse in den Stauräumen der großen 


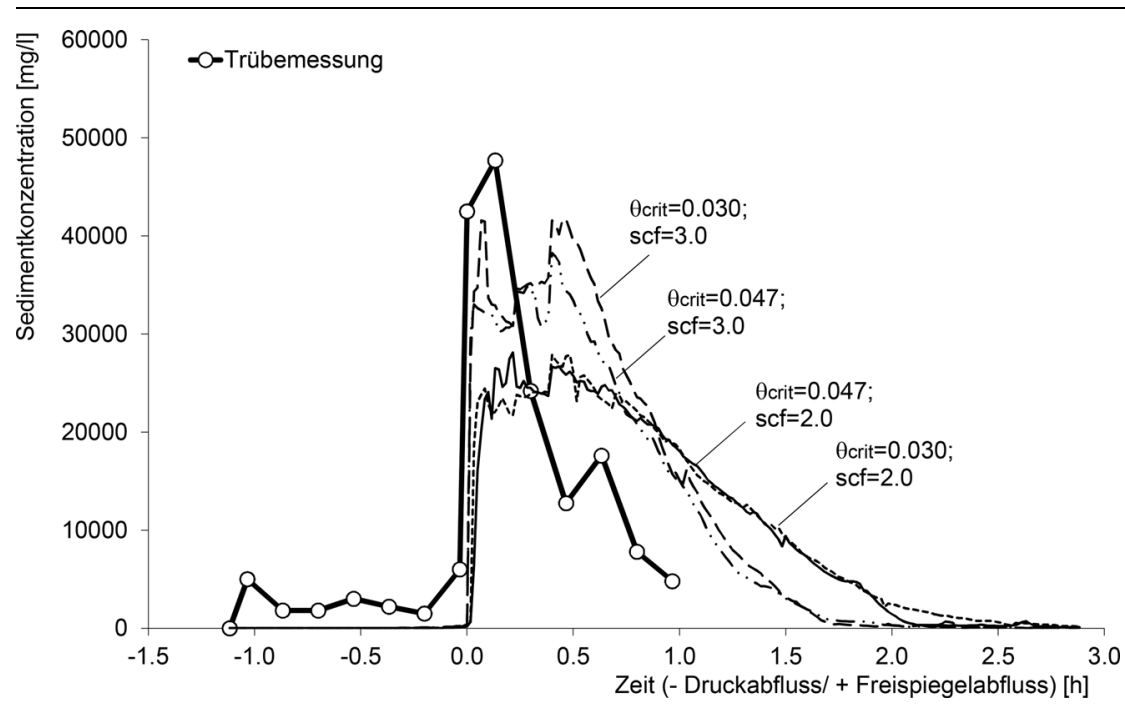

Abb. 12 Vergleich der Feststoffkonzentration aus Trübemessungen in der Unterwasserstrecke mit jenen aus den numerischen Berechnungen

Wasserfassungen sehr gut abgebildet werden. Große und grobkörnige Geschiebefrachten infolge von Hochwasserereignissen können zu lokal unnatürlichen Auflandungen führen, welche Anpassungen einzelner Modellparameter erfordern. Die Steuerung von Auflandungen und Erosionen im Bereich der Zulaufstrecke kann durch geringe Veränderungen des scf-Wertes und der dimensionslosen kritischen Sohlschubspannung im Rahmen gängiger Literaturwerte erfolgen.

- Im Bereich der Stauraumspülung ergibt sich trotz der Veränderung der Modellparameter im Rahmen der empfohlenen Literaturwerte eine Unterschätzung des Transports um den Faktor 2 bis 3. Ausgehend von der korrekten Darstellung der Erosionsprozesse im Modellversuch und den umfangreichen Naturmessungen bei einer realen Spülung, zeigt sich, dass insbesondere die Erosionsprozesse während einer Stauraumspülung mit den verwendeten Formelsätzen in den numerischen Berechnungen unbefriedigend abgebildet werden. Während eine Verfeinerung der räumlichen und zeitlichen Diskretisierung des numerischen Modells und eine Adaption der Anpassungslänge noch als vertretbare Maßnahmen für die Erhöhung des Geschiebetransportes angesehen werden können, nimmt die Erhöhung des Transports mithilfe einer linearen Skalierung (scfWert) eine Sonderstellung ein. Die erforderliche Skalierung ist abhängig vom Verlandungsvolumen, vom Spülabfluss und von der Kornzusammensetzung des Geschiebes, und stützt sich ausschließlich auf die Ergebnisse des Modellversuchs und der Naturmessungen.

- Für die Spülung der Unterwasserstrecke führen eine Reduktion der dimensionslosen kritischen Sohlschubspannung und die Vernachlässigung von Formverlusten im Gerinne durch entsprechende Definition der Rauheiten zu einer besseren Mobilisierbarkeit des Geschiebes. Zusätzlich kann eine Verstärkung des Transports durch die Erhöhung des scf-Werts erfolgen. Die verwendeten Parameter stellen Grenzwerte der Literaturgrößen dar, womit die besonderen hydraulischen Bedingungen auch während der Spülung in der Unterwasserstrecke verdeutlicht werden.

Die Ergebnisse der Untersuchungen zeigen, dass 2D-numerische Berechnungen mit einer Definition der Pa- rametersätze ohne zugrunde liegende Vergleichsgrößen aus einem Modellversuch und/oder Naturmessungen $\mathrm{zu}$ einer großen Unterschätzung der Transportleistungen führen können. Die implementierten Formelsätze sind nicht uneingeschränkt für die Bestimmung der hoch instationären Erosionsprozesse geeignet. Damit sind im Zuge der Kalibrierung teilweise signifikante manuelle Eingriffe in die Parameterwahl notwendig. Trotz dieser Modellierungsprobleme kann mit dem dargestellten Modellkonzept, welches für die 2D-numerischen Berechnungen Kalibrierungs- und Validierungsgrößen aus einem physikalischen Modellversuch und aus Naturmessungen bezieht, eine Beurteilung der Verlandungs- und Spülprozesse an den geplanten Wasserfassungen durchgeführt werden. Es konnte nachgewiesen werden, dass sowohl bei Regelbetrieb als auch bei Hochwasserereignissen die Spülungen des Stauraums und der Unterwasserstrecke erfolgreich durchgeführt werden können.

Danksagung Der Arbeitsbereich Wasserbau der Universität Innsbruck bedankt sich bei der Tiroler Wasserkraft AG für die hervorragende Zusammenarbeit, die fachlichen Diskussionen und die umfangreiche Unterstützung bei der Bearbeitung der Projekte, welche die Grundlage für diese Zusammenfassung bilden.

Open access funding provided by University of Innsbruck and Medical University of Innsbruck.

Open Access Dieser Artikel wird unter der Creative Commons Namensnennung 4.0 International Lizenz (http:// creativecommons.org/licenses/by/4. $0 /$ deed.de) veröffentlicht, welche die Nutzung, Vervielfältigung, Bearbeitung, Verbreitung und Wiedergabe in jeglichem Medium und Format erlaubt, sofern Sie den/die ursprünglichen $\mathrm{Au}$ tor(en) und die Quelle ordnungsgemäß nennen, einen Link zur Creative Commons Lizenz beifügen und angeben, ob Änderungen vorgenommen wurden. 
Arbeitsbereich Wasserbau, Universität Innsbruck (Hrsg.) (2012): Modellversuch Wasserfassung Gurgler Ache. Arbeitsbereich Wasserbau, Universität Innsbruck

Arbeitsbereich Wasserbau, Universität Innsbruck (Hrsg.) (2015a): 2D-numerische Modellierung des Geschiebetransportes an den Wasserfassungen Gurgler Ache und Venter Ache. Arbeitsbereich Wasserbau, Universität Innsbruck

Arbeitsbereich Wasserbau, Universität Innsbruck (Hrsg.) (2015b): 2D-numerische Modellierung der Stauraumspülung an der Wasserfassung Taschach am 24.07.2014. Arbeitsbereich Wasserbau, Universität Innsbruck

Brune, G. (1953): Trap efficiency of reservoirs. American Geophysical Union, Vol. 34, Nr. 3 407-418.

Gems, B. (2011): Entwicklung eines integrativen Konzeptes zur Modellierung hochwasserrelevanter Prozesse und Bewertung der Wirkung von Hochwasserschutzmaßnahmen in alpinen Talschaften - Modellanwendung auf Basis eine regionalen Betrachtungsebene am Beispiel des Ötztals in den Tiroler Alpen. Arbeitsbereich Wasserbau -Universität Innsbruck, Dissertation, 274 Seiten.
Gems, B., Achleitner S., Huttenlau M., Thieken A., Aufleger M. (2009): Flood control management for an alpine valley in Tyrol - an integrated hydrological-hydraulic approach. In: 33rd Congress of the International Association of Hydraulic Engineering \& Research (IAHR) „Wate engineering for a sustainable environment". Eigenverlag - IAHR/IWA, ISBN 978-90-78046-08-0, S. $1779-1786$.

Hunziker, R.P. (1995): Fraktionsweiser Geschiebetransport. Versuchsanstalt für Wasserbau, Hydrologie und Glaziologie der Eidgenössischen Technischen Hochschule Zürich, Mitteilung 138 Hunziker, R. P., Fardel, A., Garbani Nerini, P. (2009): Hydro_GS-2D Mehrkorn: Erweiterung des Programms HYDRO GS-2D mit einem Mehrkornmodul auf Basis des Programms MORMO Modell und Versuchsbeschreibung

Meyer-Peter, E., Müller, R. (1949): Eine Formel zur Berechnung des Geschiebetriebs. In: Schweizerische Bauzeitung 67 (1949), Nr. 3, S. 29\{32\}

Nujic, M. (2009): Hydro AS-2D - Ein zweidimensionales Strömungsmodell für die wasserwirtschaftliche Praxis - Benutzerhandbuch, 73 Seiten

Nujic, M. (2015): Hydro_FT-2D - Erweiterung zu Hydro_AS-2D zur Simulation des Stofftransports, 44 Seiten
Plörer, M., Achleitner, S., Neuner, J., Aufleger, M. (2013a): Sedimentation and flushing of alpine water intake reservoirs - 2D numerical simulation based on calibration data of a hydraulic model test. Proceedings of 2013 IAHR Congress, 2013 Tsinghua University Press, Beijing.

Plörer, M., Achleitner, S., Neuner, J., Mayer, R. Lumassegger, S., Aufleger, M. (2013b): Sedimentation and flushing of alpine water intake reservoirs, hydraulic model test. In 10th International Conference on Fluvial Sedimentology, Leeds, U.K. 14-19 July 2013.

TIWAG (2016): http://wasserkraftausbau.tiwag. at/wasserkraftausbau, aufgerufen im September, 2016

Tschada, H., Hofer, B. (1990): Der Feststoffhaushalt im Einzugsgebiet des Kaunertalkraftwerkes - Erkenntnisse aus 25-jähriger Betriebserfahrung, Tagungsbeitrag IAHS-Kongress Lausanne 1990

Umach, L. (2014): Bedeutung der Approximation von Kornverteilungskurven bei der zweidimensionalen numerischen Simulation von Geschiebetransportprozessen. Arbeitsbereich Wasserbau -Universität Innsbruck, Dissertation, 240 Seiten 